\title{
INVERSE ELECTROMAGNETIC DIFFRACTION BY BIPERIODIC DIELECTRIC GRATINGS
}

\author{
XUE JIANG AND PEIJUN LI
}

\begin{abstract}
Consider the incidence of a time-harmonic electromagnetic plane wave onto a biperiodic dielectric grating, where the surface is assumed to be a small and smooth perturbation of a plane. The diffraction is modeled as a transmission problem for Maxwell's equations in three dimensions. This paper concerns the inverse diffraction problem which is to reconstruct the grating surface from either the diffracted field or the transmitted field. A novel approach is developed to solve the challenging nonlinear and ill-posed inverse problem. The method requires only a single incident field and is realized via the fast Fourier transform. Numerical results show that it is simple, fast, and stable to reconstruct biperiodic dielectric grating surfaces with super-resolved resolution.
\end{abstract}

\section{INTRODUCTION}

Consider the diffraction of a time-harmonic electromagnetic plane incident wave by a biperiodic structure, which is called a crossed or two-dimensional grating in optical community. Given the structure and the incident field, the direct problem is to determine the diffracted field. The inverse problem is to reconstruct the grating surface from measured field. This paper concerns the latter. Diffractive gratings have been widely used in micro-optics including the design and fabrication of optical elements such as corrective lenses, antireflective interfaces, beam splitters, and sensors. Driven by the industrial applications, the diffraction grating problems have received ever-lasting attention in the engineering and applied mathematical communities [9. 40]. An introduction to this topic can be found in the monograph [42]. We refer to [7] for a comprehensive review on the mathematical modeling and computational methods for these problems.

The inverse diffraction problems have been studied extensively for one-dimensional gratings, where the structures are invariant in one direction and the model of Maxwell's equations can be simplified into the Helmholtz equation. Mathematical results, such as uniqueness and stability, are established by many researchers [5, 10, 20, 32, 36]. Computationally, a number of methods are developed [4, 16, 17, 21, 30, 31, 35]. Numerical solutions can be found in [3, 26, 28, 37] for solving general inverse surface scattering problems. There are also many work done for two-dimensional gratings, where Maxwell's equations must be considered. We refer to [6, 15, 29,38] for the existence, uniqueness, and numerical approximations of solutions for the direct problems. Mathematical studies on uniqueness can be found in [2, 18, 19, 33, 34, 44] for the inverse problems. Numerical results are very rare for the inverse problems due to the nonlinearity, ill-posedness, and large scale computation [39]. Despite a great number of work done for the inverse diffraction problems, they addressed the classical inverse scattering problems. The reconstructed resolution was limited by Rayleigh's criterion, approximately half of the incident wavelength, also known as the diffraction limit [27].

Recently, a novel approach has been developed to solve inverse surface scattering problems in various near-field imaging modalities [11-13, 25] including the inverse electromagnetic diffraction by a perfectly electrically conducting grating [8]. This work presents the first quantitative method for solving the inverse diffraction problem of Maxwell's equations with super-resolved resolution. As is known, the perfect electric conductor is an idealized material exhibiting infinite electrical conductivity and may not exist in nature. In this paper, we consider a realistic dielectric grating and the result is closer to practical applications. The more

2010 Mathematics Subject Classification. 65N21, 78A46.

Key words and phrases. Maxwell's equations, near-field imaging, biperiodic gratings, inverse diffraction.

The research of XJ was supported in part by China NSF grant 11401040 and by the Fundamental Research Funds for the Central Universities 24820152015RC17. The research of PL was partially supported by NSF DMS-1151308. 
elaborate techniques differ from the existing work because a complicated transmission problem of Maxwell's equations needs to be studied. Related work on near-field imaging may be found in [14, 23, 24, 39].

Specifically, we consider the incidence of an electromagnetic plane wave on a dielectric crossed grating, where the surface is assumed to be a small and smooth deformation of a plane. The diffraction is modeled as a transmission problem for Maxwell's equations in three dimensions. The method begins with the transformed field expansion and reduces the boundary value problem into a successive sequence of two-point boundary value problems. Dropping higher order terms in the expansion, we linearize the nonlinear inverse problem and obtain explicit reconstruction formulas for both the reflection and transmission configurations. A spectral cut-off regularization is adopted to suppress the exponential growth of the noise in the evanescent wave components, which carry high spatial frequency of the surface and contribute to the super resolution. The method requires only a single illumination with one polarization, one frequency, and one incident direction, and is realized via the fast Fourier transform. The numerical results are computed by using synthetic scattering data provided by an adaptive edge element method with a perfectly matched absorbing layer [15]. Two numerical examples, one smooth surface and one non-smooth surface, are presented to demonstrate the effectiveness of the proposed method. Careful numerical studies are carried for the influence of all the parameters on the reconstructions. The results show that the method is simple, fast, and stable to reconstruct dielectric crossed grating surfaces with subwavelength resolution.

The paper is organized as follows. In Section 2, the model problem is introduced. Section 3 presents the transformed field expansion to obtain the analytic solution of the direct problem. Explicit reconstruction formulas are derived for the inverse problem in Section 4. Numerical examples are reported in Section 5. The paper is concluded with some general remarks and direction for future work in Section 6.

\section{MODEL PROBLEM}

In this section, we define some notation and introduce a boundary value problem for the diffraction by a biperiodic dielectric grating.

2.1. Maxwell's equations. Let us first specify the problem geometry. Denote $(\rho, z) \in \mathbb{R}^{3}$, where $\rho=$ $(x, y) \in \mathbb{R}^{2}$. As seen in Figure 1, the problem may be restricted to a single period of $\Lambda=\left(\Lambda_{1}, \Lambda_{2}\right)$ in $\rho$ due to the periodicity of the structure. Let the surface in one period be described by $S=\left\{(\rho, z) \in \mathbb{R}^{3}: z=\right.$ $\left.\phi(\rho), 0<x<\Lambda_{1}, 0<y<\Lambda_{2}\right\}$, where $\phi \in C^{2}\left(\mathbb{R}^{2}\right)$ is a biperiodic grating surface function. We assume that

$$
\phi(\rho)=\delta \psi(\rho),
$$

where $\delta>0$ is a small surface deformation parameter, $\psi \in C^{2}\left(\mathbb{R}^{2}\right)$ is also a biperiodic function and describes the shape of the grating surface.

We let $S$ be embedded in the rectangular slab:

$$
\Omega=\left\{(\rho, z) \in \mathbb{R}^{3}: z_{-}<z<z_{+}\right\}=\mathbb{R}^{2} \times\left(z_{-}, z_{+}\right),
$$

where $z_{+}>0$ and $z_{-}<0$ are two constants. Hence the domain $\Omega$ is bounded by two plane surfaces $\Gamma_{ \pm}=\left\{(\rho, z) \in \mathbb{R}^{3}: z=z_{ \pm}\right\}$. Let $\Omega_{S}^{+}=\{(\rho, z): z>\phi(\rho)\}$ and $\Omega_{S}^{-}=\{(\rho, z): z<\phi(\rho)\}$ be filled with homogeneous materials which are characterized by the electric permittivity $\varepsilon_{+}$and $\varepsilon_{-}$, respectively.

Let $\left(\mathbf{E}^{\text {inc }}, \mathbf{H}^{\text {inc }}\right)$ be the incoming electromagnetc plane waves, where

$$
\mathbf{E}^{\mathrm{inc}}=\mathbf{p} e^{\mathrm{i} \kappa_{+}(\alpha \cdot \rho-\beta z)}, \quad \mathbf{H}^{\mathrm{inc}}=\left(\frac{\varepsilon_{+}}{\mu}\right)^{1 / 2} \mathbf{q} e^{\mathrm{i} \kappa_{+}(\alpha \cdot \rho-\beta z)} .
$$

Here $\kappa_{+}=\omega\left(\mu \varepsilon_{+}\right)^{1 / 2}$ is the wavenumber in $\Omega_{S}^{+}, \omega>0$ is the angular frequency, $\mu$ is the magnetic permeability and is assumed to be a positive constant everywhere, $\alpha=\left(\alpha_{1}, \alpha_{2}\right), \alpha_{1}=\sin \theta_{1} \cos \theta_{2}, \alpha_{2}=\sin \theta_{1} \sin \theta_{2}$, and $\beta=\cos \theta_{1}$, where $\theta_{1}$ and $\theta_{2}$ are the latitudinal and longitudinal incident angles, respectively, which satisfy $0 \leq \theta_{1}<\pi / 2,0 \leq \theta_{2}<2 \pi$. Denote by $\mathbf{d}=\left(\alpha_{1}, \alpha_{2},-\beta\right)$ the unit propagation direction vector. The unit polarization vectors $\mathbf{p}=\left(p_{1}, p_{2}, p_{3}\right)$ and $\mathbf{q}=\left(q_{1}, q_{2}, q_{3}\right)$ satisfy

$$
\mathbf{p} \cdot \mathbf{d}=0, \quad \mathbf{q}=\mathbf{d} \times \mathbf{p},
$$




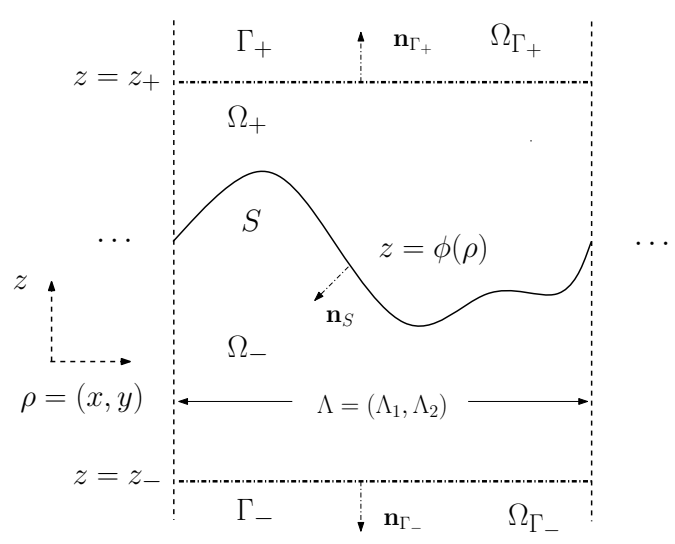

FIGURE 1. The problem geometry of a biperiodic dielectric grating.

which gives explicitly that

$$
q_{1}=\alpha_{2} p_{3}+\beta p_{2}, \quad q_{2}=-\left(\alpha_{1} p_{3}+\beta p_{1}\right), \quad q_{3}=\alpha_{1} p_{2}-\alpha_{2} p_{1} .
$$

For normal incident, i.e., $\theta_{1}=0$, we have

$$
\alpha_{1}=0, \quad \alpha_{2}=0, \quad \beta=1, \quad q_{1}=p_{2}, \quad q_{2}=-p_{1}, \quad q_{3}=0 .
$$

Hence we get from $|\mathbf{p}|=|\mathbf{q}|=1$ that

$$
p_{1}^{2}+p_{2}^{2}=1, \quad p_{3}=0 .
$$

For simplicity, we focus on the normal incidence from now on since our method requires only a single incidence. In fact, this is the most convenient way to illuminate the grating structure. The method also works for non-normal incidence with obvious modifications.

Let $\mathbf{E}^{\text {inc }}=\left(E_{1}^{\text {inc }}, E_{2}^{\text {inc }}, E_{3}^{\text {inc }}\right)$ and $\mathbf{H}^{\text {inc }}=\left(H_{1}^{\text {inc }}, H_{2}^{\text {inc }}, H_{3}^{\text {inc }}\right)$. Under the normal incidence, the incoming plane waves (2.2) reduce to

$$
E_{j}^{\mathrm{inc}}=p_{j} e^{-\mathrm{i} \kappa_{+} z}, \quad H_{j}^{\mathrm{inc}}=\left(\frac{\varepsilon_{+}}{\mu}\right)^{1 / 2} q_{j} e^{-\mathrm{i} \kappa_{+} z},
$$

which satisfy the time-harmonic Maxwell equation:

$$
\nabla \times \mathbf{E}^{\text {inc }}-\mathrm{i} \omega \mu \mathbf{H}^{\text {inc }}=0, \quad \nabla \times \mathbf{H}^{\text {inc }}+\mathrm{i} \omega \varepsilon_{+} \mathbf{E}^{\text {inc }}=0 \quad \text { in } \Omega_{S}^{+} .
$$

The time-harmonic electromagnetic waves satisfy Maxwell's equations:

$$
\nabla \times \mathbf{E}-\mathrm{i} \omega \mu \mathbf{H}=0, \quad \nabla \times \mathbf{H}+\mathrm{i} \omega \varepsilon \mathbf{E}=0 \quad \text { in } \mathbb{R}^{3},
$$

where $(\mathbf{E}, \mathbf{H})$ are the total electric and magnetic fields, and the dielectric permittivity

$$
\varepsilon= \begin{cases}\varepsilon_{+} & \text {in } \Omega_{S}^{+}, \\ \varepsilon_{-} & \text {in } \Omega_{S}^{-} .\end{cases}
$$

Motivated by uniqueness, we are interested in periodic solutions of $(\mathbf{E}, \mathbf{H})$ in $\rho$ with period $\Lambda$, i.e., $(\mathbf{E}, \mathbf{H})$ satisfy

$$
\mathbf{E}(\rho+\Lambda, z)=\mathbf{E}(\rho, z), \quad \mathbf{H}(\rho+\Lambda, z)=\mathbf{H}(\rho, z) .
$$

The total fields can be decomposed into

$$
(\mathbf{E}, \mathbf{H})=\left\{\begin{array}{cc}
\left(\mathbf{E}^{\text {inc }}, \mathbf{H}^{\text {inc }}\right)+\left(\mathbf{E}^{\mathrm{d}}, \mathbf{H}^{\mathrm{d}}\right) & \text { in } \Omega_{S}^{+}, \\
\left(\mathbf{E}^{\mathrm{t}}, \mathbf{H}^{\mathrm{t}}\right) & \text { in } \Omega_{S}^{-},
\end{array}\right.
$$

where $\left(\mathbf{E}^{\mathrm{d}}, \mathbf{H}^{\mathrm{d}}\right)$ are the diffracted fields and $\left(\mathbf{E}^{\mathrm{t}}, \mathbf{H}^{\mathrm{t}}\right)$ are the transmitted fields. They are required to satisfy the bounded outgoing wave condition. 
2.2. Transparent boundary condition. In this section, we introduce transparent boundary conditions on $\Gamma_{ \pm}$ which are equivalent to the bounded outgoing wave condition. The detailed derivation can be found in [16].

Let $n=\left(n_{1}, n_{2}\right) \in \mathbb{Z}^{2}$ and denote $\alpha_{n}=\left(\alpha_{1 n}, \alpha_{2 n}\right)$, where $\alpha_{1 n}=2 \pi n_{1} / \Lambda_{1}$ and $\alpha_{2 n}=2 \pi n_{2} / \Lambda_{2}$. For any vector field $\mathbf{u}=\left(u_{1}, u_{2}, u_{3}\right)$, denote its tangential components on $\Gamma_{ \pm}$by

$$
\mathbf{u}_{\Gamma_{ \pm}}=\mathbf{n}_{\Gamma_{ \pm}} \times\left(\mathbf{u} \times \mathbf{n}_{\Gamma_{ \pm}}\right)=\left(u_{1}\left(\rho, z_{ \pm}\right), u_{2}\left(\rho, z_{ \pm}\right), 0\right),
$$

and its tangential traces on $\Gamma_{ \pm}$by

$$
\begin{aligned}
& \mathbf{u} \times \mathbf{n}_{\Gamma_{+}}=\left(u_{2}\left(\rho, z_{+}\right),-u_{1}\left(\rho, z_{+}\right), 0\right), \\
& \mathbf{u} \times \mathbf{n}_{\Gamma_{-}}=\left(-u_{2}\left(\rho, z_{-}\right), u_{1}\left(\rho, z_{-}\right), 0\right),
\end{aligned}
$$

where $\mathbf{n}_{\Gamma_{ \pm}}=(0,0, \pm 1)$ are the unit normal vectors on $\Gamma_{ \pm}$.

For any tangential vector $\mathbf{u}\left(\rho, z_{+}\right)=\left(u_{1}\left(\rho, z_{+}\right), u_{2}\left(\rho, z_{+}\right), 0\right)$ on $\Gamma_{+}$, where $u_{j}$ are biperiodic functions in $\rho$ with period $\Lambda$, we define the capacity operator $T_{+}$:

$$
T_{+} \mathbf{u}=\left(v_{1}\left(\rho, z_{+}\right), v_{2}\left(\rho, z_{+}\right), 0\right),
$$

where $v_{j}$ are also biperiodic functions in $\rho$ with the same period $\Lambda$. Here $u_{j}$ and $v_{j}$ have the following Fourier series expansions

$$
u_{j}\left(\rho, z_{+}\right)=\sum_{n \in \mathbb{Z}^{2}} u_{j n}\left(z_{+}\right) e^{\mathrm{i} \alpha_{n} \cdot \rho}, \quad v_{j}\left(\rho, z_{+}\right)=\sum_{n \in \mathbb{Z}^{2}} v_{j n}\left(z_{+}\right) e^{\mathrm{i} \alpha_{n} \cdot \rho},
$$

and the Fourier coefficients $u_{j n}$ and $v_{j n}$ satisfy

$$
\left\{\begin{array}{l}
v_{1 n}\left(z_{+}\right)=\frac{1}{\omega \mu \beta_{n}^{+}}\left[\left(\kappa_{+}^{2}-\alpha_{2 n}^{2}\right) u_{1 n}\left(z_{+}\right)+\alpha_{1 n} \alpha_{2 n} u_{2 n}\left(z_{+}\right)\right], \\
v_{2 n}\left(z_{+}\right)=\frac{1}{\omega \mu \beta_{n}^{+}}\left[\left(\kappa_{+}^{2}-\alpha_{1 n}^{2}\right) u_{2 n}\left(z_{+}\right)+\alpha_{1 n} \alpha_{2 n} u_{1 n}\left(z_{+}\right)\right],
\end{array}\right.
$$

where

$$
\left(\beta_{n}^{+}\right)^{2}=\kappa_{+}^{2}-\left|\alpha_{n}\right|^{2} \quad \text { with } \operatorname{Im} \beta_{n}^{+}>0 .
$$

We exclude possible resonance by assuming that $\beta_{n}^{+} \neq 0$ for all $n \in \mathbb{Z}^{2}$.

Using the capacity operator (2.5), we impose a transparent boundary condition on $\Gamma_{+}$:

$$
T_{+}\left(\mathbf{E}_{\Gamma_{+}}-\mathbf{E}_{\Gamma_{+}}^{\text {inc }}\right)=\left(\mathbf{H}-\mathbf{H}^{\text {inc }}\right) \times \mathbf{n}_{\Gamma_{+}},
$$

which maps the tangential component of the scattered electric field to the tangential trace of the scattered magnetic field. Equivalently, the above boundary condition can be written as

$$
(\nabla \times \mathbf{E}) \times \mathbf{n}_{\Gamma_{+}}=\mathrm{i} \omega \mu T_{+} \mathbf{E}_{\Gamma_{+}}+\mathbf{f},
$$

where

$$
\mathbf{f}=\mathrm{i} \omega \mu\left(\mathbf{H}^{\mathrm{inc}} \times \mathbf{n}_{\Gamma_{+}}-T_{+} \mathbf{E}_{\Gamma_{+}}^{\mathrm{inc}}\right)=\left(f_{1}, f_{2}, f_{3}\right) .
$$

Recalling the incident fields (2.3) and using the boundary operator (2.5), we have explicitly that

$$
f_{j}=-2 \mathrm{i} \kappa_{+} p_{j} e^{-\mathrm{i} \kappa_{+} z_{+}} .
$$

Similarly, for any given tangential vector $\mathbf{u}\left(\rho, z_{-}\right)=\left(u_{1}\left(\rho, z_{-}\right), u_{2}\left(\rho, z_{-}\right), 0\right)$ on $\Gamma_{-}$, where $u_{j}\left(\rho, z_{-}\right)$is a biperiodic function in $\rho$ with period $\Lambda$, we define the capacity operator $T_{-}$:

$$
T_{-} \mathbf{u}=\left(v_{1}\left(\rho, z_{-}\right), v_{2}\left(\rho, z_{-}\right), 0\right),
$$

where $v_{j}$ is also a biperiodic function in $\rho$ with the same period $\Lambda$. Here $u_{j}$ and $v_{j}$ have the following Fourier series expansions

$$
u_{j}\left(\rho, z_{-}\right)=\sum_{n \in \mathbb{Z}^{2}} u_{j n}\left(z_{-}\right) e^{\mathrm{i} \alpha_{n} \cdot \rho}, \quad v_{j}\left(\rho, z_{-}\right)=\sum_{n \in \mathbb{Z}^{2}} v_{j n}\left(z_{-}\right) e^{\mathrm{i} \alpha_{n} \cdot \rho},
$$


and the Fourier coefficients $u_{j n}$ and $v_{j n}$ satisfy

$$
\left\{\begin{array}{l}
v_{1 n}\left(z_{-}\right)=\frac{1}{\omega \mu \beta_{n}^{-}}\left[\left(\kappa_{-}^{2}-\alpha_{2 n}^{2}\right) u_{1 n}\left(z_{-}\right)+\alpha_{1 n} \alpha_{2 n} u_{2 n}\left(z_{-}\right)\right], \\
v_{2 n}\left(z_{-}\right)=\frac{1}{\omega \mu \beta_{n}^{-}}\left[\left(\kappa_{-}^{2}-\alpha_{1 n}^{2}\right) u_{2 n}\left(z_{-}\right)+\alpha_{1 n} \alpha_{2 n} u_{1 n}\left(z_{-}\right)\right],
\end{array}\right.
$$

where $\kappa_{-}=\omega\left(\mu \varepsilon_{-}\right)^{1 / 2}$ is the wavenumber in $\Omega_{S}^{-}$and

$$
\left(\beta_{n}^{-}\right)^{2}=\kappa_{-}^{2}-\left|\alpha_{n}\right|^{2} \quad \text { with } \operatorname{Im} \beta_{n}^{-}>0 .
$$

Here we also assume that $\beta_{n}^{-} \neq 0$ for all $n \in \mathbb{Z}^{2}$.

Based on (2.8), a transparent boundary condition may be proposed on $\Gamma_{-}$:

$$
T_{-} \mathbf{E}_{\Gamma_{-}}=\mathbf{H} \times \mathbf{n}_{\Gamma_{-}},
$$

which is equivalent to

$$
(\nabla \times \mathbf{E}) \times \mathbf{n}_{\Gamma_{-}}=\mathrm{i} \omega \mu T_{-} \mathbf{E}_{\Gamma_{-}} .
$$

2.3. Transmission problem. Taking curl on both sides of (2.4), we may eliminate the magnetic field and obtain a decoupled equation for the electric field:

$$
\nabla \times(\nabla \times \mathbf{E})-\kappa^{2} \mathbf{E}=0 \quad \text { in } \Omega,
$$

where the wavenumber

$$
\kappa= \begin{cases}\kappa_{+} & \text {in } \Omega_{S}^{+}, \\ \kappa_{-} & \text {in } \Omega_{S}^{-} .\end{cases}
$$

Denote $\Omega_{+}=\Omega_{S}^{+} \cap \Omega=\left\{(\rho, z): \phi(\rho)<z<z_{+}\right\}$and $\Omega_{-}=\Omega_{S}^{-} \cap \Omega=\left\{(\rho, z): z_{-}<z<\phi(\rho)\right\}$. Let $\mathbf{E}^{+}$and $\mathbf{E}^{-}$be the restriction of $\mathbf{E}$ in $\Omega_{+}$and $\Omega_{-}$, respectively, i.e., $\mathbf{E}^{ \pm}=\left.\mathbf{E}\right|_{\Omega_{ \pm}}$. It is useful to have an equivalent scalar form of (2.11) when applying the transformed field expansion. Denote $\mathbf{E}^{ \pm}=$ $\left(E_{1}^{ \pm}, E_{2}^{ \pm}, E_{3}^{ \pm}\right)$. We may reformulate (2.11) into the Helmholtz equation:

$$
\Delta E_{j}^{ \pm}+\kappa_{ \pm}^{2} E_{j}^{ \pm}=0 \quad \text { in } \Omega_{ \pm} .
$$

The transparent boundary conditions $(2.7)$ and $(2.10)$ can be written as

$$
\left\{\begin{array}{l}
\partial_{z} E_{1}^{+}-\partial_{x} E_{3}^{+}=\mathrm{i} \omega \mu H_{1}^{+}+f_{1}, \\
\partial_{z} E_{2}^{+}-\partial_{y} E_{3}^{+}=\mathrm{i} \omega \mu H_{2}^{+}+f_{2},
\end{array}\right.
$$

and

$$
\left\{\begin{array}{l}
\partial_{z} E_{1}^{-}-\partial_{x} E_{3}^{-}=-\mathrm{i} \omega \mu H_{1}^{-}, \\
\partial_{z} E_{2}^{-}-\partial_{y} E_{3}^{-}=-\mathrm{i} \omega \mu H_{2}^{-},
\end{array}\right.
$$

where the Fourier coefficients of the periodic functions $H_{1}^{ \pm}$and $H_{2}^{ \pm}$are given by

$$
\left\{\begin{array}{l}
H_{1 n}^{ \pm}\left(z_{ \pm}\right)=\frac{1}{\omega \mu \beta_{n}^{ \pm}}\left[\left(\kappa_{ \pm}^{2}-\alpha_{2 n}^{2}\right) E_{1 n}^{ \pm}\left(z_{ \pm}\right)+\alpha_{1 n} \alpha_{2 n} E_{2 n}^{ \pm}\left(z_{ \pm}\right)\right], \\
H_{2 n}^{ \pm}\left(z_{ \pm}\right)=\frac{1}{\omega \mu \beta_{n}^{ \pm}}\left[\left(\kappa_{ \pm}^{2}-\alpha_{1 n}^{2}\right) E_{2 n}^{ \pm}\left(z_{ \pm}\right)+\alpha_{1 n} \alpha_{2 n} E_{1 n}^{ \pm}\left(z_{ \pm}\right)\right] .
\end{array}\right.
$$

Here $E_{1 n}^{ \pm}\left(z_{ \pm}\right)$and $E_{2 n}^{ \pm}\left(z_{ \pm}\right)$are the Fourier coefficients of the periodic electric field $E_{1}^{ \pm}\left(\rho, z_{ \pm}\right)$and $E_{2}^{ \pm}\left(\rho, z_{ \pm}\right)$, respectively.

The continuity conditions are needed to reformulate the boundary value problem into a transmission problem. It is known that the tangential traces of the electric and magnetic fields are continuous across the grating surface, i.e.,

$$
\mathbf{E}^{+} \times \mathbf{n}_{S}=\mathbf{E}^{-} \times \mathbf{n}_{S}, \quad \mathbf{H}^{+} \times \mathbf{n}_{S}=\mathbf{H}^{-} \times \mathbf{n}_{S}, \quad z=\phi(\rho),
$$


where $\mathbf{n}_{S}=\left(\phi_{x}, \phi_{y},-1\right)$ is the normal vector on $S$ pointing from $\Omega_{S}^{+}$to $\Omega_{S}^{-}$. Explicitly, we have the continuity conditions

$$
\left\{\begin{array}{l}
E_{2}^{+}+\phi_{y} E_{3}^{+}=E_{2}^{-}+\phi_{y} E_{3}^{-}, \\
E_{1}^{+}+\phi_{x} E_{3}^{+}=E_{1}^{-}+\phi_{x} E_{3}^{-},
\end{array}\right.
$$

and

$$
\left\{\begin{array}{c}
\left(\partial_{z} E_{1}^{+}-\partial_{x} E_{3}^{+}\right)+\phi_{y}\left(\partial_{x} E_{2}^{+}-\partial_{y} E_{1}^{+}\right) \\
=\left(\partial_{z} E_{1}^{-}-\partial_{x} E_{3}^{-}\right)+\phi_{y}\left(\partial_{x} E_{2}^{-}-\partial_{y} E_{1}^{-}\right) \\
\left(\partial_{y} E_{3}^{+}-\partial_{z} E_{2}^{+}\right)+\phi_{x}\left(\partial_{x} E_{2}^{+}-\partial_{y} E_{1}^{+}\right) \\
=\left(\partial_{y} E_{3}^{-}-\partial_{z} E_{2}^{-}\right)+\phi_{x}\left(\partial_{x} E_{2}^{-}-\partial_{y} E_{1}^{-}\right)
\end{array}\right.
$$

The transparent boundary conditions (2.13), (2.14) and the continuity conditions (2.15) and (2.16) are not enough to determine the fields $\mathbf{E}_{j}^{ \pm}$. Additional information can be obtained from the divergence free conditions

$$
\partial_{x} E_{1}^{ \pm}+\partial_{y} E_{2}^{ \pm}+\partial_{z} E_{3}^{ \pm}=0 \quad \text { in } \Omega_{ \pm}
$$

Given the grating surface function $\phi(\rho)$, the direct problem is to determine the fields $\mathbf{E}_{j}^{ \pm}$. This work is focused on the inverse problem, which is to reconstruct the grating surface function $\phi(\rho)$ from the tangential traces of the total field measured at either $\Gamma_{+}$, i.e., $\mathbf{E}\left(\rho, z_{+}\right) \times \mathbf{n}_{\Gamma_{+}}=\left(E_{1}\left(\rho, z_{+}\right), E_{2}\left(\rho, z_{+}\right), 0\right)$ called the reflection configuration, or $\Gamma_{-}$, i.e., $\mathbf{E}\left(\rho, z_{-}\right) \times \mathbf{n}_{\Gamma_{-}}=\left(-E_{1}\left(\rho, z_{-}\right), E_{2}\left(\rho, z_{-}\right), 0\right)$ called the transmission configuration. In particular, we are interested in the inverse problem in near-field regime where the measurement distance $\left|z_{ \pm}\right|$is much smaller than the wavelength $\lambda=2 \pi / \kappa_{+}$of the incident field.

\section{TRANSFORMED FIELD EXPANSION}

In this section, we introduce the transformed field expansion to analytically derive the solution for the direct problem. We refer to [22,41] for solving the direct surface scattering problems by using the transformed field expansion and related boundary perturbation method.

3.1. Change of variables. Consider the change of variables:

$$
\tilde{x}=x, \quad \tilde{y}=y, \quad \tilde{z}=z_{+}\left(\frac{z-\phi}{z_{+}-\phi}\right), \quad \phi<z<z_{+},
$$

and

$$
\tilde{x}=x, \quad \tilde{y}=y, \quad \tilde{z}=z_{-}\left(\frac{z-\phi}{z_{-}-\phi}\right), \quad z_{-}<z<\phi,
$$

which maps the domain $\Omega_{+}$and $\Omega_{-}$into rectangular slabs $D_{+}=\left\{(\tilde{\rho}, \tilde{z}) \in \mathbb{R}^{3}: 0<\tilde{z}<z_{+}\right\}$and $D_{-}=\left\{(\tilde{\rho}, \tilde{z}) \in \mathbb{R}^{3}: z_{-}<z<0\right\}$, respectively.

We seek to restate the diffractive grating problem in the new coordinate. Introduce a new function $\tilde{\mathbf{E}}^{ \pm}=\left(\tilde{E}_{1}^{ \pm}, \tilde{E}_{2}^{ \pm}, \tilde{E}_{3}^{ \pm}\right)$and let $\tilde{E}_{j}^{ \pm}(\tilde{x}, \tilde{y}, \tilde{z})=E_{j}^{ \pm}(x, y, z)$ under the transformation. After tedious but straightforward calculations, it can be verified from (2.12) that the total electric field, upon dropping the tilde, satisfies the equation

$$
\begin{aligned}
c_{1}^{ \pm} \frac{\partial^{2} E_{j}^{ \pm}}{\partial x^{2}} & +c_{1}^{ \pm} \frac{\partial^{2} E_{j}^{ \pm}}{\partial y^{2}}+c_{2}^{ \pm} \frac{\partial^{2} E_{j}^{ \pm}}{\partial z^{2}}-c_{3}^{ \pm} \frac{\partial^{2} E_{j}^{ \pm}}{\partial x \partial z} \\
& -c_{4}^{ \pm} \frac{\partial^{2} E_{j}^{ \pm}}{\partial y \partial z}-c_{5}^{ \pm} \frac{\partial E_{j}^{ \pm}}{\partial z}+\kappa_{ \pm}^{2} c_{1}^{ \pm} E_{j}^{ \pm}=0 \quad \text { in } D_{ \pm}
\end{aligned}
$$


where

$$
\begin{aligned}
& c_{1}^{ \pm}=\left(z_{ \pm}-\phi\right)^{2}, \\
& c_{2}^{ \pm}=\left(\phi_{x}^{2}+\phi_{y}^{2}\right)\left(z_{ \pm}-z\right)^{2}+z_{ \pm}^{2}, \\
& c_{3}^{ \pm}=2 \phi_{x}\left(z_{ \pm}-z\right)\left(z_{ \pm}-\phi\right), \\
& c_{4}^{ \pm}=2 \phi_{y}\left(z_{ \pm}-z\right)\left(z_{ \pm}-\phi\right), \\
& c_{5}^{ \pm}=\left(z_{ \pm}-z\right)\left[\left(\phi_{x x}+\phi_{y y}\right)\left(z_{ \pm}-\phi\right)+2\left(\phi_{x}^{2}+\phi_{y}^{2}\right)\right] .
\end{aligned}
$$

The transparent boundary conditions $(2.13)$ and $(2.14)$ reduce to

$$
\left\{\begin{array}{l}
\left(\frac{z_{+}}{z_{+}-\phi}\right) \partial_{z} E_{1}^{+}-\partial_{x} E_{3}^{+}=\mathrm{i} \omega \mu H_{1}^{+}+f_{1}, \\
\left(\frac{z_{+}}{z_{+}-\phi}\right) \partial_{z} E_{2}^{+}-\partial_{y} E_{3}^{+}=\mathrm{i} \omega \mu H_{2}^{+}+f_{2},
\end{array}\right.
$$

and

$$
\left\{\begin{array}{l}
\left(\frac{z_{-}}{z_{-}-\phi}\right) \partial_{z} E_{1}^{-}-\partial_{x} E_{3}^{-}=-\mathrm{i} \omega \mu H_{1}^{-} \\
\left(\frac{z_{-}}{z_{-}-\phi}\right) \partial_{z} E_{2}^{-}-\partial_{y} E_{3}^{-}=-\mathrm{i} \omega \mu H_{2}^{-}
\end{array}\right.
$$

The continuity conditions (2.15) and (2.16) are changed to

$$
\left\{\begin{array}{l}
E_{2}^{+}+\phi_{y} E_{3}^{+}=E_{2}^{-}+\phi_{y} E_{3}^{-}, \\
E_{1}^{+}+\phi_{x} E_{3}^{+}=E_{1}^{-}+\phi_{x} E_{3}^{-},
\end{array}\right.
$$

and

$$
\left\{\begin{array}{r}
\left(\frac{z_{+}}{z_{+}-\phi}\right)\left[\phi_{x} \partial_{z} E_{3}^{+}-\phi_{x} \phi_{y} \partial_{z} E_{2}^{+}+\left(1+\phi_{y}^{2}\right) \partial_{z} E_{1}^{+}\right] \\
-\left(\partial_{x} E_{3}^{+}-\phi_{y} \partial_{x} E_{2}^{+}+\phi_{y} \partial_{y} E_{1}^{+}\right) \\
=\left(\frac{z_{-}}{z_{-}-\phi}\right)\left[\phi_{x} \partial_{z} E_{3}^{-}-\phi_{x} \phi_{y} \partial_{z} E_{2}^{-}+\left(1+\phi_{y}^{2}\right) \partial_{z} E_{1}^{-}\right] \\
-\left(\partial_{x} E_{3}^{-}-\phi_{y} \partial_{x} E_{2}^{-}+\phi_{y} \partial_{y} E_{1}^{-}\right) \\
\left(\frac{z_{+}}{z_{+}-\phi}\right)\left[\phi_{y} \partial_{z} E_{3}^{+}+\left(1+\phi_{x}^{2}\right) \partial_{z} E_{2}^{+}-\phi_{x} \phi_{y} \partial_{z} E_{1}^{+}\right] \\
-\left(\partial_{y} E_{3}^{+}+\phi_{x} \partial_{x} E_{2}^{+}-\phi_{x} \partial_{y} E_{1}^{+}\right) \\
=\left(\frac{z_{-}}{z_{-}-\phi}\right)\left[\phi_{y} \partial_{z} E_{3}^{-}+\left(1+\phi_{x}^{2}\right) \partial_{z} E_{2}^{-}-\phi_{x} \phi_{y} \partial_{z} E_{1}^{-}\right] \\
-\left(\partial_{y} E_{3}^{-}+\phi_{x} \partial_{x} E_{2}^{-}-\phi_{x} \partial_{y} E_{1}^{-}\right) .
\end{array}\right.
$$

The divergence free condition (2.17) becomes

$$
\begin{aligned}
\partial_{x} E_{1}^{ \pm}+\partial_{y} E_{2}^{ \pm}- & \left(\frac{z_{ \pm}-z}{z_{ \pm}-\phi}\right)\left(\phi_{x} \partial_{z} E_{1}^{ \pm}+\phi_{y} \partial_{z} E_{2}^{ \pm}\right) \\
& +\left(\frac{z_{ \pm}}{z_{ \pm}-\phi}\right) \partial_{z} E_{3}^{ \pm}=0 \text { in } D_{ \pm} .
\end{aligned}
$$


3.2. Power series. Recalling $\phi=\delta \psi$ in (2.1), we use a classical boundary perturbation argument and consider a formal expansion of $E_{j}^{ \pm}$in a power series of $\delta$ :

$$
E_{j}^{ \pm}(\rho, z ; \delta)=\sum_{k=0}^{\infty} E_{j}^{ \pm(k)}(\rho, z) \delta^{k} .
$$

Substituting $\phi=\delta \psi$ and the power series expansion (3.7) into $c_{j}^{ \pm}$and (3.1), we may derive a recursion equation for $E_{j}^{ \pm(k)}$ :

$$
\Delta E_{j}^{ \pm(k)}+\kappa_{ \pm}^{2} E_{j}^{ \pm(k)}=F_{j}^{ \pm(k)} \text { in } D_{ \pm},
$$

where the nonhomogeneous term

$$
\begin{aligned}
F_{j}^{ \pm(k)} & =\frac{2 \psi}{z_{ \pm}} \frac{\partial^{2} E_{j}^{ \pm(k-1)}}{\partial x^{2}}+\frac{2 \psi}{z_{ \pm}} \frac{\partial^{2} E_{j}^{ \pm(k-1)}}{\partial y^{2}}+\frac{2\left(z_{ \pm}-z\right) \psi_{x}}{z_{ \pm}} \frac{\partial^{2} E_{j}^{ \pm(k-1)}}{\partial x \partial z} \\
& +\frac{2\left(z_{ \pm}-z\right) \psi_{y}}{z_{ \pm}} \frac{\partial^{2} E_{j}^{ \pm(k-1)}}{\partial y \partial z}+\frac{\left(z_{ \pm}-z\right)\left(\psi_{x x}+\psi_{y y}\right)}{z_{ \pm}} \frac{\partial E_{j}^{ \pm(k-1)}}{\partial z}+\frac{2 \kappa_{ \pm}^{2} \psi}{z_{ \pm}} E_{j}^{ \pm(k-1)} \\
& -\frac{\psi^{2}}{z_{ \pm}^{2}} \frac{\partial^{2} E_{j}^{ \pm(k-2)}}{\partial x^{2}}-\frac{\psi^{2}}{z_{ \pm}^{2}} \frac{\partial^{2} E_{j}^{ \pm(k-2)}}{\partial y^{2}}-\frac{\left(z_{ \pm}-z\right)^{2}\left(\psi_{x}^{2}+\psi_{y}^{2}\right)}{z_{ \pm}^{2}} \frac{\partial^{2} E_{j}^{ \pm(k-2)}}{\partial z^{2}} \\
& -\frac{2 \psi \psi_{x}\left(z_{ \pm}-z\right)}{z_{ \pm}^{2}} \frac{\partial^{2} E_{j}^{ \pm(k-2)}}{\partial x \partial z}-\frac{2 \psi \psi_{y}\left(z_{ \pm}-z\right)}{z_{ \pm}^{2}} \frac{\partial^{2} E_{j}^{ \pm(k-2)}}{\partial y \partial z} \\
& +\frac{\left(z_{ \pm}-z\right)\left[2\left(\psi_{x}^{2}+\psi_{y}^{2}\right)-\psi\left(\psi_{x x}+\psi_{y y}\right)\right]}{z_{ \pm}^{2}} \frac{\partial E_{j}^{ \pm(k-2)}}{\partial z}-\frac{\kappa_{ \pm}^{2} \psi^{2}}{z_{ \pm}^{2}} E_{j}^{ \pm(k-2)} .
\end{aligned}
$$

Here $\psi_{x}=\partial_{x} \psi(x, y)$ and $\psi_{y}=\partial_{y} \psi(x, y)$ are the partial derivatives.

Substituting (3.7) into the transparent boundary conditions (3.2) and (3.3), we obtain

$$
\left\{\begin{array}{l}
\partial_{z} E_{1}^{+(k)}-\partial_{x} E_{3}^{+(k)}=\mathrm{i} \omega \mu H_{1}^{+(k)}-f_{1}^{+(k)}, \\
\partial_{z} E_{2}^{+(k)}-\partial_{y} E_{3}^{+(k)}=\mathrm{i} \omega \mu H_{2}^{+(k)}-f_{2}^{+(k)},
\end{array}\right.
$$

and

$$
\left\{\begin{array}{l}
\partial_{z} E_{1}^{-(k)}-\partial_{x} E_{3}^{-(k)}=-\mathrm{i} \omega \mu H_{1}^{-(k)}-f_{1}^{-(k)}, \\
\partial_{z} E_{2}^{-(k)}-\partial_{y} E_{3}^{-(k)}=-\mathrm{i} \omega \mu H_{2}^{-(k)}-f_{2}^{-(k)},
\end{array}\right.
$$

where

$$
\begin{aligned}
& f_{1}^{+(0)}=-f_{1}, f_{1}^{+(1)}=\frac{\psi}{z_{+}} \partial_{z} E_{1}^{+(0)}, f_{1}^{+(k)}=\frac{\psi}{z_{+}}\left(\partial_{x} E_{3}^{+(k-1)}+\mathrm{i} \omega \mu H_{1}^{+(k-1)}\right), \\
& f_{2}^{+(0)}=-f_{2}, f_{2}^{+(1)}=\frac{\psi}{z_{+}} \partial_{z} E_{2}^{+(1)}, f_{2}^{+(k)}=\frac{\psi}{z_{+}}\left(\partial_{y} E_{3}^{+(k-1)}+\mathrm{i} \omega \mu H_{2}^{+(k-1)}\right),
\end{aligned}
$$

and

$$
\begin{aligned}
& f_{1}^{-(0)}=0, f_{1}^{-(1)}=\frac{\psi}{z_{-}} \partial_{z} E_{1}^{-(0)}, f_{1}^{-(k)}=\frac{\psi}{z_{-}}\left(\partial_{x} E_{3}^{-(k-1)}-\mathrm{i} \omega \mu H_{1}^{-(k-1)}\right), \\
& f_{2}^{-(0)}=0, f_{2}^{-(1)}=\frac{\psi}{z_{-}} \partial_{z} E_{2}^{-(0)}, f_{2}^{-(k)}=\frac{\psi}{z_{-}}\left(\partial_{y} E_{3}^{-(k-1)}-\mathrm{i} \omega \mu H_{2}^{-(k-1)}\right) .
\end{aligned}
$$


Here the Fourier coefficients of $H_{1}^{ \pm(k)}\left(\rho, z_{ \pm}\right)$and $H_{2}^{ \pm(k)}\left(\rho, z_{ \pm}\right)$are

$$
\left\{\begin{array}{l}
H_{1 n}^{ \pm(k)}\left(z_{ \pm}\right)=\frac{1}{\omega \mu \beta_{n}^{ \pm}}\left[\left(\kappa_{ \pm}^{2}-\alpha_{2 n}^{2}\right) E_{1 n}^{ \pm(k)}\left(z_{ \pm}\right)+\alpha_{1 n} \alpha_{2 n} E_{2 n}^{ \pm(k)}\left(z_{ \pm}\right)\right] \\
H_{2 n}^{ \pm(k)}\left(z_{ \pm}\right)=\frac{1}{\omega \mu \beta_{n}^{ \pm}}\left[\left(\kappa_{ \pm}^{2}-\alpha_{1 n}^{2}\right) E_{2 n}^{ \pm(k)}\left(z_{ \pm}\right)+\alpha_{1 n} \alpha_{2 n} E_{1 n}^{ \pm(k)}\left(z_{ \pm}\right)\right] .
\end{array}\right.
$$

Here $E_{1 n}^{ \pm(k)}\left(z_{ \pm}\right)$and $E_{2 n}^{ \pm(k)}\left(z_{ \pm}\right)$are the Fourier coefficients of $E_{1}^{ \pm(k)}\left(\rho, z_{ \pm}\right)$and $E_{1}^{ \pm(k)}\left(\rho, z_{ \pm}\right)$, respectively.

Plugging (3.7) into the jump conditions (3.4) and (3.5) yields

$$
\left\{\begin{array}{l}
E_{2}^{+(k)}+\psi_{y} E_{3}^{+(k-1)}=E_{2}^{-(k)}+\psi_{y} E_{3}^{-(k-1)}, \\
E_{1}^{+(k)}+\psi_{x} E_{3}^{+(k-1)}=E_{1}^{-(k)}+\psi_{x} E_{3}^{-(k-1)},
\end{array}\right.
$$

and

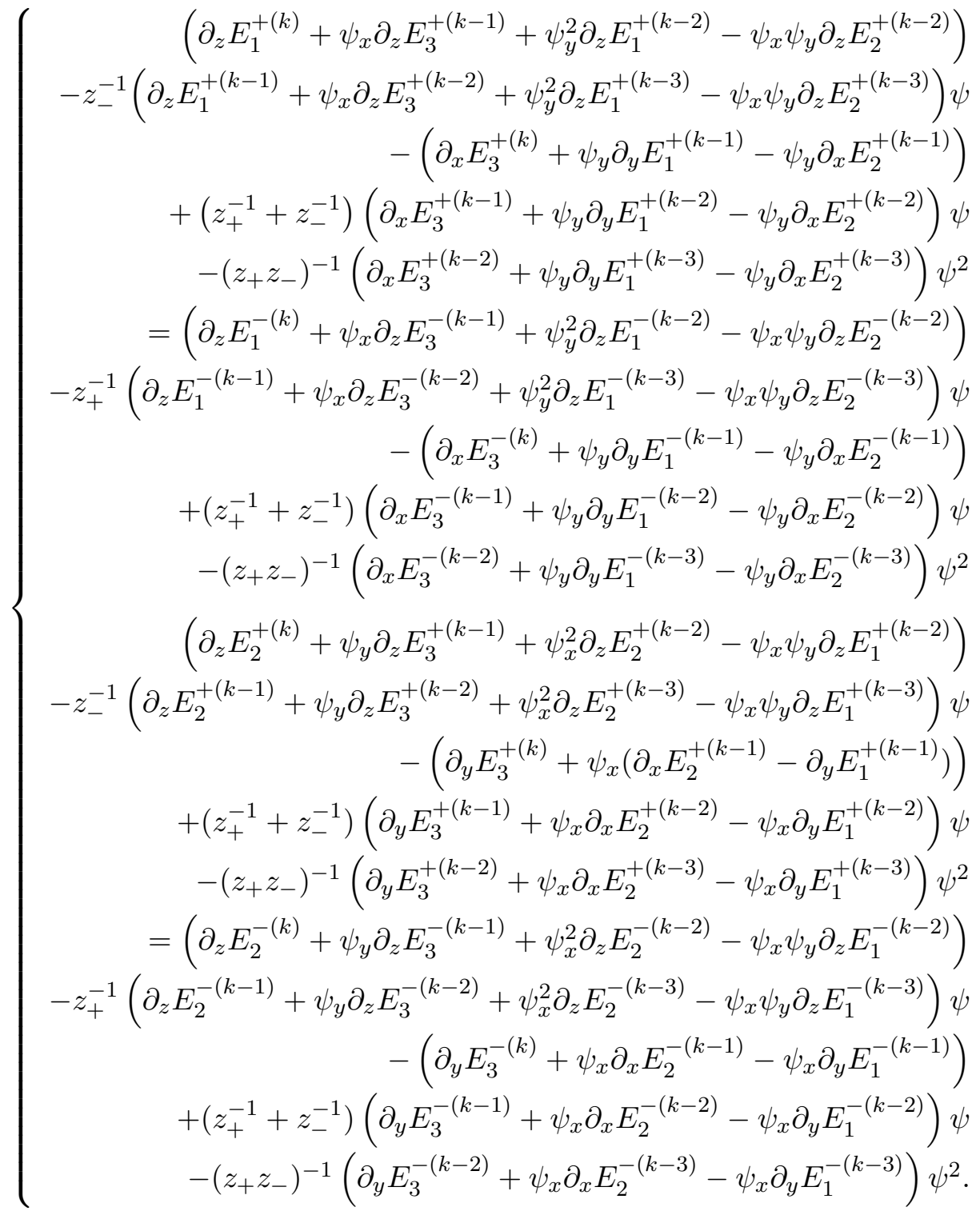

Substituting (3.7) into the divergence free condition (3.6) yields

$$
\partial_{x} E_{1}^{ \pm(k)}+\partial_{y} E_{2}^{ \pm(k)}+\partial_{z} E_{3}^{ \pm(k)}=g^{ \pm(k)} \text { in } D_{ \pm},
$$


where

$$
\begin{aligned}
w^{ \pm(k)}= & \frac{\psi}{z_{ \pm}}\left(\partial_{x} E_{1}^{ \pm(k-1)}+\partial_{y} E_{2}^{ \pm(k-1)}\right) \\
& +\left(\frac{z_{ \pm}-z}{z_{ \pm}}\right)\left(\psi_{x} \partial_{z} E_{1}^{ \pm(k-1)}+\psi_{y} \partial_{z} E_{2}^{ \pm(k-1)}\right) .
\end{aligned}
$$

3.3. Zeroth order. Recalling the recurrence relation (3.8) and letting $k=0$, we have

$$
\Delta E_{j}^{ \pm(0)}+\kappa_{ \pm}^{2} E_{j}^{ \pm(0)}=0 \text { in } D_{ \pm} .
$$

The transparent boundary conditions (3.9) and (3.10) becomes

$$
\left\{\begin{array}{l}
\partial_{z} E_{1}^{+(0)}\left(\rho, z_{+}\right)-\partial_{x} E_{3}^{+(0)}\left(\rho, z_{+}\right)=\mathrm{i} \omega \mu H_{1}^{+(0)}\left(\rho, z_{+}\right)+f_{1}(\rho), \\
\partial_{z} E_{2}^{+(0)}\left(\rho, z_{+}\right)-\partial_{y} E_{3}^{+(0)}\left(\rho, z_{+}\right)=\mathrm{i} \omega \mu H_{2}^{+(0)}\left(\rho, z_{+}\right)+f_{2}(\rho),
\end{array}\right.
$$

and

$$
\left\{\begin{array}{l}
\partial_{z} E_{1}^{-(0)}\left(\rho, z_{-}\right)-\partial_{x} E_{3}^{-(0)}\left(\rho, z_{-}\right)=-\mathrm{i} \omega \mu H_{1}^{-(0)}\left(\rho, z_{-}\right), \\
\partial_{z} E_{2}^{-(0)}\left(\rho, z_{-}\right)-\partial_{y} E_{3}^{-(0)}\left(\rho, z_{-}\right)=-\mathrm{i} \omega \mu H_{2}^{-(0)}\left(\rho, z_{-}\right) .
\end{array}\right.
$$

The jump conditions (3.11) and (3.12) reduce to

$$
E_{2}^{+(0)}(\rho, 0)=E_{2}^{-(0)}(\rho, 0), \quad E_{1}^{+(0)}(\rho, 0)=E_{1}^{-(0)}(\rho, 0),
$$

and

$$
\left\{\begin{array}{l}
\partial_{z} E_{1}^{+(0)}(\rho, 0)-\partial_{x} E_{3}^{+(0)}(\rho, 0)=\partial_{z} E_{1}^{-(0)}(\rho, 0)-\partial_{x} E_{3}^{-(0)}(\rho, 0), \\
\partial_{z} E_{2}^{+(0)}(\rho, 0)-\partial_{y} E_{3}^{+(0)}(\rho, 0)=\partial_{z} E_{2}^{-(0)}(\rho, 0)-\partial_{y} E_{3}^{-(0)}(\rho, 0) .
\end{array}\right.
$$

The divergence free condition (3.13) reduces to

$$
\partial_{x} E_{1}^{ \pm(0)}+\partial_{y} E_{2}^{ \pm(0)}+\partial_{z} E_{3}^{ \pm(0)}=0 \text { in } D_{ \pm} .
$$

Since $E_{j}^{ \pm(0)}(\rho, z)$ and $f_{j}$ are periodic functions of $\rho$ with period $\Lambda$, they have the following Fourier expansion

$$
E_{j}^{ \pm(0)}(\rho, z)=\sum_{n \in \mathbb{Z}^{2}} E_{j n}^{ \pm(0)}(z) e^{\mathrm{i} \alpha_{n} \cdot \rho}, \quad f_{j}(\rho)=\sum_{n \in \mathbb{Z}^{2}} f_{j n} e^{\mathrm{i} \alpha_{n} \cdot \rho}
$$

where

$$
f_{j n}=\left\{\begin{array}{cc}
-2 \mathrm{i} \kappa_{+} p_{j} e^{-\mathrm{i} \kappa_{+} z_{+}} & \text {for } \\
0 & \text { for } n \neq 0,
\end{array}\right.
$$

Plugging (3.20) into (3.14)-3.19), we obtain an ordinary differential equation

$$
\frac{\mathrm{d}^{2} E_{j n}^{ \pm(0)}(z)}{\mathrm{d} z^{2}}+\left(\beta_{n}^{ \pm}\right)^{2} E_{j n}^{ \pm(0)}(z)=0,
$$

together with the boundary conditions at $z=z_{+}$:

$$
\left\{\begin{array}{l}
E_{1 n}^{+(0)^{\prime}}-\mathrm{i} \alpha_{1 n} E_{3 n}^{+(0)}=\frac{\mathrm{i}}{\beta_{n}^{+}}\left[\left(\kappa_{+}^{2}-\alpha_{2 n}^{2}\right) E_{1 n}^{+(0)}+\alpha_{1 n} \alpha_{2 n} E_{2 n}^{+(0)}\right]+f_{1 n} \\
E_{2 n}^{+(0)^{\prime}}-\mathrm{i} \alpha_{2 n} E_{3 n}^{+(0)}=\frac{\mathrm{i}}{\beta_{n}^{+}}\left[\left(\kappa_{+}^{2}-\alpha_{1 n}^{2}\right) E_{2 n}^{+(0)}+\alpha_{1 n} \alpha_{2 n} E_{1 n}^{+(0)}\right]+f_{2 n} \\
E_{3 n}^{+(0)^{\prime}}+\mathrm{i} \alpha_{1 n} E_{1 n}^{+(0)}+\mathrm{i} \alpha_{2 n} E_{2 n}^{+(0)}=0
\end{array}\right.
$$


and the boundary conditions at $z=z_{-}$:

$$
\left\{\begin{array}{l}
E_{1 n}^{-(0)^{\prime}}-\mathrm{i} \alpha_{1 n} E_{3 n}^{-(0)}=-\frac{\mathrm{i}}{\beta_{n}^{-}}\left[\left(\kappa_{-}^{2}-\alpha_{2 n}^{2}\right) E_{1 n}^{-(0)}+\alpha_{1 n} \alpha_{2 n} E_{2 n}^{-(0)}\right], \\
E_{2 n}^{-(0)^{\prime}}-\mathrm{i} \alpha_{2 n} E_{3 n}^{-(0)}=-\frac{\mathrm{i}}{\beta_{n}^{-}}\left[\left(\kappa_{-}^{2}-\alpha_{1 n}^{2}\right) E_{2 n}^{-(0)}+\alpha_{1 n} \alpha_{2 n} E_{1 n}^{-(0)}\right], \\
E_{3 n}^{-(0)^{\prime}}+\mathrm{i} \alpha_{1 n} E_{1 n}^{-(0)}+\mathrm{i} \alpha_{2 n} E_{2 n}^{-(0)}=0,
\end{array}\right.
$$

and the jump conditions at $z=0$ :

$$
E_{2 n}^{+(0)}=E_{2 n}^{-(0)}, \quad E_{1 n}^{+(0)}=E_{1 n}^{-(0)},
$$

and

$$
\left\{\begin{array}{l}
E_{1 n}^{+(0)^{\prime}}-\mathrm{i} \alpha_{1 n} E_{3 n}^{+(0)}=E_{1 n}^{-(0)^{\prime}}-\mathrm{i} \alpha_{1 n} E_{3 n}^{-(0)}, \\
E_{2 n}^{+(0)^{\prime}}-\mathrm{i} \alpha_{2 n} E_{3 n}^{+(0)}=E_{2 n}^{-(0)^{\prime}}-\mathrm{i} \alpha_{2 n} E_{3 n}^{-(0)} .
\end{array}\right.
$$

It can be verified that the general solutions of the homogeneous second order equations (3.21) are

$$
E_{j n}^{ \pm(0)}(z)=A_{j n}^{ \pm} e^{\mathrm{i} \beta_{n}^{ \pm} z}+B_{j n}^{ \pm} e^{-\mathrm{i} \beta_{n}^{ \pm} z}
$$

where $A_{j n}^{ \pm}, B_{j n}^{ \pm} \in \mathbb{C}$ are to be determined. Substituting (3.26) into the boundary conditions (3.22) and (3.23), and the jump conditions (3.24) and (3.25), we may deduce that

$$
A_{j 0}^{+(0)}=r p_{j}, \quad B_{j 0}^{+(0)}=p_{j}, \quad A_{j 0}^{-(0)}=0, \quad B_{j 0}^{-(0)}=t p_{j},
$$

and $A_{j n}^{ \pm}=B_{j n}^{ \pm}=A_{j n}^{ \pm}=B_{j n}^{ \pm}=0$ for $n \neq 0$, where

$$
r=\frac{\kappa_{+}-\kappa_{-}}{\kappa_{+}+\kappa_{-}} \quad \text { and } \quad t=\frac{2 \kappa_{+}}{\kappa_{+}+\kappa_{-}}
$$

are known as the reflection coefficient and the transmission coefficient, respectively. Hence we find the analytic expressions for the zeroth order terms:

$$
\left\{\begin{array}{l}
E_{j}^{+(0)}(\rho, z)=p_{j}\left(e^{-\mathrm{i} \kappa_{+} z}+r e^{\mathrm{i} \kappa_{+} z}\right), \\
E_{j}^{-(0)}(\rho, z)=p_{j} t e^{-\mathrm{i} \kappa_{-} z} .
\end{array}\right.
$$

Clearly, the zeroth order terms consist of the incident wave, the reflected wave, and the transmitted wave, which come from the diffraction of an electromagnetic plane wave by a planar surface.

3.4. First order. In this section, we derive analytic expressions of the first order terms, and particularly a connection between their Fourier coefficients and the Fourier coefficient of the grating profile.

Taking $k=1$ in (3.8) yields

$$
\Delta E_{j}^{ \pm(1)}+\kappa^{2} E_{j}^{ \pm(1)}=F_{j}^{ \pm(1)} \text { in } D_{ \pm}
$$

where

$$
\begin{aligned}
F_{j}^{ \pm(1)} & =\frac{2 \psi}{z_{ \pm}} \frac{\partial^{2} E_{j}^{ \pm(0)}}{\partial x^{2}}+\frac{2 \psi}{z_{ \pm}} \frac{\partial^{2} E_{j}^{ \pm(0)}}{\partial y^{2}}+\frac{2\left(z_{ \pm}-z\right) \psi_{x}}{z_{ \pm}} \frac{\partial^{2} E_{j}^{ \pm(0)}}{\partial x \partial z}+\frac{2\left(z_{ \pm}-z\right) \psi_{y}}{z_{ \pm}} \frac{\partial^{2} E_{j}^{ \pm(0)}}{\partial y \partial z} \\
& +\frac{\left(z_{ \pm}-z\right)\left(\psi_{x x}+\psi_{y y}\right)}{z_{ \pm}} \frac{\partial E_{j}^{ \pm(0)}}{\partial z}+\frac{2 \kappa_{ \pm}^{2} \psi}{z_{ \pm}} E_{j}^{ \pm(0)}
\end{aligned}
$$


It follows from the explicit expression of the zeroth order term (3.27) that we have

$$
\begin{aligned}
F_{j}^{+(1)}(\rho, z) & =\frac{2 \kappa_{+}^{2} p_{j}}{z_{+}}\left(e^{-\mathrm{i} \kappa_{+} z}+r e^{\mathrm{i} \kappa_{+} z}\right) \psi \\
& -\frac{\mathrm{i} \kappa_{+} p_{j}\left(z_{+}-z\right)}{z_{+}}\left(e^{-\mathrm{i} \kappa_{+} z}-r e^{\mathrm{i} \kappa_{+} z}\right)\left(\psi_{x x}+\psi_{y y}\right)
\end{aligned}
$$

and

$$
F_{j}^{-(1)}(\rho, z)=\frac{2 \kappa_{-}^{2} p_{j}}{z_{-}} t e^{-\mathrm{i} \kappa_{-} z} \psi-\frac{\mathrm{i} \kappa_{-} p_{j}\left(z_{-}-z\right)}{z_{-}} t e^{-\mathrm{i} \kappa_{-} z}\left(\psi_{x x}+\psi_{y y}\right) .
$$

The transparent boundary conditions (3.9) and (3.10) become

$$
\left\{\begin{array}{l}
\partial_{z} E_{1}^{+(1)}\left(\rho, z_{+}\right)-\partial_{x} E_{3}^{+(1)}\left(\rho, z_{+}\right)=\mathrm{i} \omega \mu H_{1}^{+(1)}\left(\rho, z_{+}\right)-f_{1}^{+(1)}(\rho) \\
\partial_{z} E_{2}^{+(1)}\left(\rho, z_{+}\right)-\partial_{y} E_{3}^{+(1)}\left(\rho, z_{+}\right)=\mathrm{i} \omega \mu H_{2}^{+(1)}\left(\rho, z_{+}\right)-f_{2}^{+(1)}(\rho)
\end{array}\right.
$$

and

$$
\left\{\begin{array}{l}
\partial_{z} E_{1}^{-(1)}\left(\rho, z_{-}\right)-\partial_{x} E_{3}^{-(1)}\left(\rho, z_{-}\right)=-\mathrm{i} \omega \mu H_{1}^{-(1)}\left(\rho, z_{-}\right)-f_{1}^{-(1)}(\rho), \\
\partial_{z} E_{2}^{-(1)}\left(\rho, z_{-}\right)-\partial_{y} E_{3}^{-(1)}\left(\rho, z_{-}\right)=-\mathrm{i} \omega \mu H_{2}^{-(1)}\left(\rho, z_{-}\right)-f_{2}^{-(1)}(\rho),
\end{array}\right.
$$

where we have from (3.27) that

$$
\begin{aligned}
& f_{j}^{+(1)}(\rho)=\frac{\psi}{z_{+}} \partial_{z} E_{j}^{+(0)}\left(\rho, z_{+}\right)=-\frac{\mathrm{i} \kappa_{+} p_{j}}{z_{+}}\left(e^{-\mathrm{i} \kappa_{+} z_{+}}-r e^{\mathrm{i} \kappa_{+} z_{+}}\right) \psi, \\
& f_{j}^{-(1)}(\rho)=\frac{\psi}{z_{-}} \partial_{z} E_{j}^{-(0)}\left(\rho, z_{-}\right)=-\frac{\mathrm{i} \kappa_{-} p_{j}}{z_{-}} t e^{-\mathrm{i} \kappa_{-} z_{-}} \psi .
\end{aligned}
$$

The jump conditions (3.11) and (3.12) reduce to

$$
\left\{\begin{array}{l}
E_{2}^{+(1)}+\psi_{y} E_{3}^{+(0)}=E_{2}^{-(1)}+\psi_{y} E_{3}^{-(0)} \\
E_{1}^{+(1)}+\psi_{x} E_{3}^{+(0)}=E_{1}^{-(1)}+\psi_{x} E_{3}^{-(0)}
\end{array}\right.
$$

and

$$
\left\{\begin{array}{c}
\partial_{z} E_{1}^{+(1)}-\frac{\psi}{z_{-}} \partial_{z} E_{1}^{+(0)}-\partial_{x} E_{3}^{+(1)}=\partial_{z} E_{1}^{-(1)}-\frac{\psi}{z_{+}} \partial_{z} E_{1}^{-(0)}-\partial_{x} E_{3}^{-(1)}, \\
\partial_{z} E_{2}^{+(1)}-\frac{\psi}{z_{-}} \partial_{z} E_{2}^{+(0)}-\partial_{y} E_{3}^{+(1)}=\partial_{z} E_{2}^{-(1)}-\frac{\psi}{z_{+}} \partial_{z} E_{2}^{-(0)}-\partial_{y} E_{3}^{-(1)},
\end{array}\right.
$$

which gives after substitution of (3.27) that

$$
E_{2}^{+(1)}=E_{2}^{-(1)}, \quad E_{1}^{+(1)}=E_{1}^{-(1)},
$$

and

$$
\left\{\begin{array}{l}
\partial_{z} E_{1}^{+(1)}-\partial_{x} E_{3}^{+(1)}+\frac{\mathrm{i} \kappa_{+} p_{1}}{z_{-}}(1-r) \psi=\partial_{z} E_{1}^{-(1)}-\partial_{x} E_{3}^{-(1)}+\frac{\mathrm{i} \kappa_{-} p_{1}}{z_{+}} t \psi \\
\partial_{z} E_{2}^{+(1)}-\partial_{y} E_{3}^{+(1)}+\frac{\mathrm{i} \kappa_{+} p_{2}}{z_{-}}(1-r) \psi=\partial_{z} E_{2}^{-(1)}-\partial_{y} E_{3}^{-(1)}+\frac{\mathrm{i} \kappa_{-} p_{2}}{z_{+}} t \psi
\end{array}\right.
$$

The divergence free condition 3.13) reduces to

$$
\partial_{x} E_{1}^{ \pm(1)}+\partial_{y} E_{2}^{ \pm(1)}+\partial_{z} E_{3}^{ \pm(1)}=g^{ \pm(1)} \text { in } D_{ \pm},
$$

where

$$
g^{ \pm(1)}(\rho, z)=\frac{\psi}{z_{ \pm}}\left(\partial_{x} E_{1}^{ \pm(0)}+\partial_{y} E_{2}^{ \pm(0)}\right)+\left(\frac{z_{ \pm}-z}{z_{ \pm}}\right)\left(\psi_{x} \partial_{z} E_{1}^{ \pm(0)}+\psi_{y} \partial_{z} E_{2}^{ \pm(0)}\right)
$$


Using (3.27, we get

$$
g^{+(1)}(\rho, z)=-\frac{\mathrm{i} \kappa_{+}\left(z_{+}-z\right)}{z_{+}}\left(e^{-\mathrm{i} \kappa_{+} z}-r e^{\mathrm{i} \kappa_{+} z}\right)\left(p_{1} \psi_{x}+p_{2} \psi_{y}\right)
$$

and

$$
g^{-(1)}(\rho, z)=-\frac{\mathrm{i} \kappa_{-}\left(z_{-}-z\right)}{z_{-}} t e^{-\mathrm{i} \kappa_{-} z}\left(p_{1} \psi_{x}+p_{2} \psi_{y}\right)
$$

Since $\psi(\rho), E_{j}^{ \pm(1)}(\rho, z)$, and $F_{j}^{ \pm(1)}(\rho, z)$ are periodic functions of $\rho$ with period $\Lambda$, they have the following Fourier expansions

$$
\begin{aligned}
\psi(\rho) & =\sum_{n \in \mathbb{Z}^{2}} \psi_{n} e^{\mathrm{i} \alpha_{n} \cdot \rho}, \\
E_{j}^{ \pm(1)}(\rho, z) & =\sum_{n \in \mathbb{Z}^{2}} E_{j n}^{ \pm(1)}(z) e^{\mathrm{i} \alpha_{n} \cdot \rho}, \\
F_{j}^{ \pm(1)}(\rho, z) & =\sum_{n \in \mathbb{Z}^{2}} F_{j n}^{ \pm(1)}(z) e^{\mathrm{i} \alpha_{n} \cdot \rho}
\end{aligned}
$$

where

$$
\begin{aligned}
F_{j n}^{+(1)}(z)= & {\left[\frac{2 \kappa_{+}^{2} p_{j}}{z_{+}}\left(e^{-\mathrm{i} \kappa_{+} z}+r e^{\mathrm{i} \kappa_{+} z}\right)\right.} \\
& \left.+\frac{\mathrm{i} \kappa_{+} p_{j}\left(z_{+}-z\right)}{z_{+}}\left(\alpha_{1 n}^{2}+\alpha_{2 n}^{2}\right)\left(e^{-\mathrm{i} \kappa_{+} z}-r e^{\mathrm{i} \kappa_{+} z}\right)\right] \psi_{n}
\end{aligned}
$$

and

$$
F_{j n}^{-(1)}(z)=\left[\frac{2 \kappa_{-}^{2} p_{j}}{z_{-}} t e^{-\mathrm{i} \kappa_{-} z}+\frac{\mathrm{i} \kappa_{-} p_{j}\left(z_{-}-z\right)}{z_{-}}\left(\alpha_{1 n}^{2}+\alpha_{2 n}^{2}\right) t e^{-\mathrm{i} \kappa_{-} z}\right] \psi_{n} .
$$

Plugging the above Fourier expansions into (3.28) and using (3.29)-(3.33), we derive an ordinary differential equation

$$
\frac{\mathrm{d}^{2} E_{j n}^{ \pm(1)}(z)}{\mathrm{d} z^{2}}+\left(\beta_{n}^{ \pm}\right)^{2} E_{j n}^{ \pm(1)}(z)=F_{j n}^{ \pm(1)}(z),
$$

together with the boundary conditions at $z=z_{+}$:

$$
\left\{\begin{array}{l}
E_{1 n}^{+(1)^{\prime}}-\mathrm{i} \alpha_{1 n} E_{3 n}^{+(1)}=\frac{\mathrm{i}}{\beta_{n}^{+}}\left[\left(\kappa_{+}^{2}-\alpha_{2 n}^{2}\right) E_{1 n}^{+(1)}+\alpha_{1 n} \alpha_{2 n} E_{2 n}^{+(1)}\right]-f_{1 n}^{+(1)}, \\
E_{2 n}^{+(1)^{\prime}}-\mathrm{i} \alpha_{2 n} E_{3 n}^{+(1)}=\frac{\mathrm{i}}{\beta_{n}^{+}}\left[\left(\kappa_{+}^{2}-\alpha_{1 n}^{2}\right) E_{2 n}^{+(1)}+\alpha_{1 n} \alpha_{2 n} E_{1 n}^{+(1)}\right]-f_{2 n}^{+(1)} \\
E_{3 n}^{+(1)^{\prime}}+\mathrm{i} \alpha_{1 n} E_{1 n}^{+(1)}+\mathrm{i} \alpha_{2 n} E_{2 n}^{+(1)}=0
\end{array}\right.
$$

and the boundary conditions at $z=z_{-}$:

$$
\left\{\begin{array}{l}
E_{1 n}^{-(1)^{\prime}}-\mathrm{i} \alpha_{1 n} E_{3 n}^{-(1)}=-\frac{\mathrm{i}}{\beta_{n}^{-}}\left[\left(\kappa_{-}^{2}-\alpha_{2 n}^{2}\right) E_{1 n}^{-(1)}+\alpha_{1 n} \alpha_{2 n} E_{2 n}^{-(1)}\right]-f_{1 n}^{-(1)}, \\
E_{2 n}^{-(1)^{\prime}}-\mathrm{i} \alpha_{2 n} E_{3 n}^{-(1)}=-\frac{\mathrm{i}}{\beta_{n}^{-}}\left[\left(\kappa_{-}^{2}-\alpha_{1 n}^{2}\right) E_{2 n}^{-(1)}+\alpha_{1 n} \alpha_{2 n} E_{1 n}^{-(1)}\right]-f_{2 n}^{-(1)} \\
E_{3 n}^{-(1)^{\prime}}+\mathrm{i} \alpha_{1 n} E_{1 n}^{-(1)}+\mathrm{i} \alpha_{2 n} E_{2 n}^{-(1)}=0
\end{array}\right.
$$


where $f_{j n}^{ \pm(1)}$ are the Fourier coefficients of $f_{j}^{ \pm(1)}(\rho)$. Explicitly, we have

$$
\begin{aligned}
& f_{j n}^{+(1)}=-\frac{\mathrm{i} \kappa_{+} p_{j}}{z_{+}}\left(e^{-\mathrm{i} \kappa_{+} z_{+}}-r e^{\mathrm{i} \kappa_{+} z_{+}}\right) \psi_{n}, \\
& f_{j n}^{-(1)}=-\frac{\mathrm{i} \kappa_{-} p_{j}}{z_{-}} t e^{-\mathrm{i} \kappa_{-} z_{-}} \psi_{n} .
\end{aligned}
$$

Using the identity $\kappa_{+}(1-r)=\kappa_{-} t$, we may reduce the jump conditions (3.31) and (3.32) to

$$
E_{2 n}^{+(1)}=E_{2 n}^{-(1)}, \quad E_{1 n}^{+(1)}=E_{1 n}^{-(1)},
$$

and

$$
\left\{\begin{array}{c}
E_{1 n}^{+(1)^{\prime}}-\mathrm{i} \alpha_{1 n} E_{3 n}^{+(1)}=E_{1 n}^{-(1)^{\prime}}-\mathrm{i} \alpha_{1 n} E_{3 n}^{-(1)}+\mathrm{i} \kappa_{-} t p_{1}\left(z_{+}^{-1}-z_{-}^{-1}\right) \psi_{n}, \\
E_{2 n}^{+(1)^{\prime}}-\mathrm{i} \alpha_{2 n} E_{3 n}^{+(1)}=E_{2 n}^{-(1)^{\prime}}-\mathrm{i} \alpha_{2 n} E_{3 n}^{-(1)}+\mathrm{i} \kappa_{-} t p_{2}\left(z_{+}^{-1}-z_{-}^{-1}\right) \psi_{n}
\end{array}\right.
$$

Based on the same identity $\kappa_{+}(1-r)=\kappa_{-} t$, we may obtain two more conditions at $z=0$ from (3.33):

$$
\left\{\begin{array}{l}
E_{3 n}^{+(1)^{\prime}}+\mathrm{i} \alpha_{1 n} E_{1 n}^{+(1)}+\mathrm{i} \alpha_{2 n} E_{2 n}^{+(1)}=\kappa_{-} t\left(\alpha_{1 n} p_{1}+\alpha_{2 n} p_{2}\right) \psi_{n}, \\
E_{3 n}^{-(1)^{\prime}}+\mathrm{i} \alpha_{1 n} E_{1 n}^{-(1)}+\mathrm{i} \alpha_{2 n} E_{2 n}^{-(1)}=\kappa_{-} t\left(\alpha_{1 n} p_{1}+\alpha_{2 n} p_{2}\right) \psi_{n} .
\end{array}\right.
$$

It follows from (3.34) that the general solutions of $E_{j n}^{ \pm(1)}$ consist of the general solution for the corresponding homogeneous equation and a particular solution for the non-homogeneous equation:

$$
E_{j n}^{+(1)}(z)=A_{j n}^{+} e^{\mathrm{i} \beta_{n}^{+} z}+B_{j n}^{+} e^{-\mathrm{i} \beta_{n}^{+} z}-\frac{\mathrm{i} \kappa_{+} p_{j}}{z_{+}}\left(z_{+}-z\right)\left(e^{-\mathrm{i} \kappa_{+} z}-r e^{\mathrm{i} \kappa_{+} z}\right) \psi_{n}
$$

and

$$
E_{j n}^{-(1)}(z)=A_{j n}^{-} e^{\mathrm{i} \beta_{n}^{-} z}+B_{j n}^{-} e^{-\mathrm{i} \beta_{n}^{-} z}-\frac{\mathrm{i} \kappa_{-} p_{j}}{z_{-}}\left(z_{-}-z\right) t e^{-\mathrm{i} \kappa_{-} z} \psi_{n}
$$

Plugging (3.40) and (3.41) into (3.35) and (3.36), and using the identity $\kappa_{ \pm}^{2}=\left(\beta_{n}^{ \pm}\right)^{2}+\alpha_{1 n}^{2}+\alpha_{2 n}^{2}$, we obtain

$$
\left\{\begin{array}{c}
\alpha_{1 n}^{2} A_{1 n}^{+}+\left[2\left(\beta_{n}^{+}\right)^{2}+\alpha_{1 n}^{2}\right] e^{-2 \mathrm{i} \beta_{n}^{+} z_{+}} B_{1 n}^{+}+\alpha_{1 n} \alpha_{2 n} A_{2 n}^{+} \\
\quad+\alpha_{1 n} \alpha_{2 n} e^{-2 \mathrm{i} \beta_{n}^{+} z_{+}} B_{2 n}^{+}=-\alpha_{1 n} \beta_{n}^{+} A_{3 n}^{+}-\alpha_{1 n} \beta_{n}^{+} e^{-2 \mathrm{i} \beta_{n}^{+} z_{+}} B_{3 n}^{+}, \\
\alpha_{2 n}^{2} A_{2 n}^{+}+\left[2\left(\beta_{n}^{+}\right)^{2}+\alpha_{2 n}^{2}\right] e^{-2 \mathrm{i} \beta_{n}^{+} z_{+}} B_{2 n}^{+}+\alpha_{1 n} \alpha_{2 n} A_{1 n}^{+} \\
\quad+\alpha_{1 n} \alpha_{2 n} e^{-2 \mathrm{i} \beta_{n}^{+} z_{+}} B_{1 n}^{+}=-\alpha_{2 n} \beta_{n} A_{3 n}^{+}-\alpha_{2 n} \beta_{n}^{+} e^{-2 \mathrm{i} \beta_{n}^{+} z_{+}} B_{3 n}^{+}, \\
\alpha_{1 n} A_{1 n}^{+}+\alpha_{1 n} e^{-2 \mathrm{i} \beta_{n}^{+} z_{+}} B_{1 n}^{+}+\alpha_{2 n} A_{2 n}^{+}+\alpha_{2 n} e^{-2 \mathrm{i} \beta_{n}^{+} z_{+}} B_{2 n}^{+} \\
=-\beta_{n}^{+} A_{3 n}^{+}+\beta_{n}^{+} e^{-2 \mathrm{i} \beta_{n}^{+} z_{+}} B_{3 n}^{+} .
\end{array}\right.
$$

and

$$
\left\{\begin{array}{l}
{\left[2\left(\beta_{n}^{-}\right)^{2}+\alpha_{1 n}^{2}\right] A_{1 n}^{-}+\alpha_{1 n}^{2} e^{-2 \mathrm{i} \beta_{n}^{-} z_{-}} B_{1 n}^{-}+\alpha_{1 n} \alpha_{2 n} A_{2 n}^{-}} \\
\quad+\alpha_{1 n} \alpha_{2 n} e^{-2 \mathrm{i} \beta_{n}^{-} z_{-}} B_{2 n}^{-}=\alpha_{1 n} \beta_{n}^{-} A_{3 n}^{-}+\alpha_{1 n} \beta_{n}^{-} e^{-2 \mathrm{i} \beta_{n}^{-} z_{-}} B_{3 n}^{-}, \\
{\left[2\left(\beta_{n}^{-}\right)^{2}+\alpha_{2 n}^{2}\right] A_{2 n}^{-}+\alpha_{2 n}^{2} e^{-2 \mathrm{i} \beta_{n}^{-} z_{-}} B_{2 n}^{-}+\alpha_{1 n} \alpha_{2 n} A_{1 n}^{-}} \\
\quad+\alpha_{1 n} \alpha_{2 n} e^{-2 \mathrm{i} \beta_{n}^{-} z_{-}} B_{1 n}^{-}=\alpha_{2 n} \beta_{n}^{-} A_{3 n}^{-}+\alpha_{2 n} \beta_{n}^{-} e^{-2 \mathrm{i} \beta_{n}^{-} z_{-}} B_{3 n}^{-}, \\
\alpha_{1 n} A_{1 n}^{-}+\alpha_{1 n} e^{-2 \mathrm{i} \beta_{n}^{-} z_{-}} B_{1 n}^{-}+\alpha_{2 n} A_{2 n}^{-}+\alpha_{2 n} e^{-2 \mathrm{i} \beta_{n}^{-} z_{-}} B_{2 n}^{-} \\
=-\beta_{n}^{-} A_{3 n}^{-}+\beta_{n}^{-} e^{-2 \mathrm{i} \beta_{n}^{-} z_{-}} B_{3 n}^{-} .
\end{array}\right.
$$


Multiplying individually $\alpha_{1 n}$ and $\alpha_{2 n}$ on both sides of the third equation in (3.42) and (3.43), and subtracting them from the first and second equation, respectively, we get

$$
B_{1 n}^{+}=-\frac{\alpha_{1 n}}{\beta_{n}^{+}} B_{3 n}^{+}, \quad B_{2 n}^{+}=-\frac{\alpha_{2 n}}{\beta_{n}^{+}} B_{3 n}^{+}, \quad A_{1 n}^{-}=\frac{\alpha_{1 n}}{\beta_{n}^{-}} A_{3 n}^{-}, \quad A_{2 n}^{-}=\frac{\alpha_{2 n}}{\beta_{n}^{-}} A_{3 n}^{-} .
$$

Substituting (3.44) into the third equations in (3.42) and (3.43) yields

$$
\left\{\begin{array}{c}
\alpha_{1 n} A_{1 n}^{+}+\alpha_{2 n} A_{2 n}^{+}=\frac{\kappa_{+}^{2}}{\beta_{n}^{+}} e^{-2 \mathrm{i} \beta_{n}^{+} z_{+}} B_{3 n}^{+}-\beta_{n}^{+} A_{3 n}^{+}, \\
\alpha_{1 n} B_{1 n}^{-}+\alpha_{2 n} B_{2 n}^{-}=\beta_{n}^{-} B_{3 n}^{-}-\frac{\kappa_{-}^{2}}{\beta_{n}^{-}} e^{2 \mathrm{i} \beta_{n}^{-} z_{-}} A_{3 n}^{-} .
\end{array}\right.
$$

Substituting (3.44), (3.40), and (3.41) into (3.39), we get

$$
\left\{\begin{array}{c}
\alpha_{1 n} A_{1 n}^{+}+\alpha_{2 n} A_{2 n}^{+}=\frac{\kappa_{+}^{2}}{\beta_{n}^{+}} B_{3 n}^{+}-\beta_{n}^{+} A_{3 n}^{+} \\
\alpha_{1 n} B_{1 n}^{-}+\alpha_{2 n} B_{2 n}^{-}=\beta_{n}^{-} B_{3 n}^{-}-\frac{\kappa_{-}^{2}}{\beta_{n}^{-}} A_{3 n}^{-} .
\end{array}\right.
$$

Combining (3.44)-(3.46) gives

$$
B_{1 n}^{+}=B_{2 n}^{+}=B_{3 n}^{+}=0 \quad \text { and } \quad A_{1 n}^{-}=A_{2 n}^{-}=A_{3 n}^{-}=0 .
$$

Plugging (3.47), (3.40), and (3.41) into (3.37) and (3.38), we obtain

$$
\left\{\begin{array}{c}
A_{1 n}^{+}-B_{1 n}^{-}=0, \\
\beta_{n}^{+} A_{1 n}^{+}+\beta_{n}^{-} B_{1 n}^{-}=\alpha_{1 n}\left(A_{3 n}^{+}-B_{3 n}^{-}\right)-2 \mathrm{i} \kappa_{+}\left(\kappa_{+}-\kappa_{-}\right) p_{1} \psi_{n},
\end{array}\right.
$$

and

$$
\left\{\begin{array}{c}
A_{2 n}^{+}-B_{2 n}^{-}=0, \\
\beta_{n}^{+} A_{2 n}^{+}+\beta_{n}^{-} B_{2 n}^{-}=\alpha_{2 n}\left(A_{3 n}^{+}-B_{3 n}^{-}\right)-2 \mathrm{i} \kappa_{+}\left(\kappa_{+}-\kappa_{-}\right) p_{2} \psi_{n} .
\end{array}\right.
$$

Upon solving (3.48) and (3.49), we have

$$
\left\{\begin{array}{l}
A_{1 n}^{+}=B_{1 n}^{-}=\left(\beta_{n}^{+}+\beta_{n}^{-}\right)^{-1}\left[\alpha_{1 n}\left(A_{3 n}^{+}-B_{3 n}^{-}\right)-2 \mathrm{i} \kappa_{+}\left(\kappa_{+}-\kappa_{-}\right) p_{1} \psi_{n}\right], \\
A_{2 n}^{+}=B_{2 n}^{-}=\left(\beta_{n}^{+}+\beta_{n}^{-}\right)^{-1}\left[\alpha_{2 n}\left(A_{3 n}^{+}-B_{3 n}^{-}\right)-2 \mathrm{i} \kappa_{+}\left(\kappa_{+}-\kappa_{-}\right) p_{2} \psi_{n}\right] .
\end{array}\right.
$$

Substituting (3.50) into (3.46) and noting (3.47), we may derive after tedious calculations that

$$
\left\{\begin{array}{l}
A_{3 n}^{+}=\frac{2 \mathrm{i} \beta_{n}^{-} \kappa_{+}\left(\kappa_{+}-\kappa_{-}\right)\left(p_{1} \alpha_{1 n}+p_{2} \alpha_{2 n}\right)}{\left(\beta_{n}^{+}+\beta_{n}^{-}\right)\left(\alpha_{1 n}^{2}+\alpha_{2 n}^{2}+\beta_{n}^{+} \beta_{n}^{-}\right)} \psi_{n}, \\
B_{3 n}^{-}=-\frac{2 \mathrm{i} \beta_{n}^{+} \kappa_{+}\left(\kappa_{+}-\kappa_{-}\right)\left(p_{1} \alpha_{1 n}+p_{2} \alpha_{2 n}\right)}{\left(\beta_{n}^{+}+\beta_{n}^{-}\right)\left(\alpha_{1 n}^{2}+\alpha_{2 n}^{2}+\beta_{n}^{+} \beta_{n}^{-}\right)} \psi_{n} .
\end{array}\right.
$$

Plugging (3.51) into (3.50) yields

$$
A_{1 n}^{+}=B_{1 n}^{-}=C_{1 n} \psi_{n}, \quad A_{2 n}^{+}=B_{2 n}^{-}=C_{2 n} \psi_{n},
$$

where

$$
\left\{\begin{array}{c}
C_{1 n}=\frac{2 \mathrm{i} \kappa_{+}\left(\kappa_{+}-\kappa_{-}\right)}{\left(\beta_{n}^{+}+\beta_{n}^{-}\right)}\left[\frac{\alpha_{1 n}\left(p_{1} \alpha_{1 n}+p_{2} \alpha_{2 n}\right)}{\left(\alpha_{1 n}^{2}+\alpha_{2 n}^{2}+\beta_{n}^{+} \beta_{n}^{-}\right)}-p_{1}\right], \\
C_{2 n}=\frac{2 \mathrm{i} \kappa_{+}\left(\kappa_{+}-\kappa_{-}\right)}{\left(\beta_{n}^{+}+\beta_{n}^{-}\right)}\left[\frac{\alpha_{2 n}\left(p_{1} \alpha_{1 n}+p_{2} \alpha_{2 n}\right)}{\left(\alpha_{1 n}^{2}+\alpha_{2 n}^{2}+\beta_{n}^{+} \beta_{n}^{-}\right)}-p_{2}\right] .
\end{array}\right.
$$

Substituting (3.47) and (3.52) into (3.40) and (3.41), and evaluating at $z_{+}$and $z_{-}$, respectively, we obtain

$$
E_{j n}^{+(1)}\left(z_{+}\right)=C_{j n} e^{\mathrm{i} \beta_{n}^{+} z_{+}} \psi_{n}, \quad E_{j n}^{-(1)}\left(z_{-}\right)=C_{j n} e^{-\mathrm{i} \beta_{n}^{-} z_{-}} \psi_{n} .
$$




\section{RECONSTRUCTION FORMULA}

In this section, we present an explicit reconstruction formula for the inverse grating surface problem by using the scattering data.

Assume that the noisy data takes the form

$$
E_{j}^{ \pm \gamma}\left(\rho, z_{ \pm}\right)=E_{j}^{ \pm}\left(\rho, z_{ \pm}\right)+\mathcal{O}(\gamma)
$$

where $E_{j}^{ \pm}\left(\rho, z_{ \pm}\right), j=1,2$ are the exact data and $\gamma$ is the noise level.

Evaluating the power series (3.7) at $z=z_{ \pm}$and replacing $E_{j}^{ \pm}\left(\rho, z_{ \pm}\right)$with the noisy data $E_{j}^{ \pm \gamma}\left(\rho, z_{ \pm}\right)$, we have

$$
E_{j}^{ \pm \gamma}\left(\rho, z_{ \pm}\right)=E_{j}^{ \pm(0)}\left(\rho, z_{ \pm}\right)+\delta E_{j}^{ \pm(1)}\left(\rho, z_{ \pm}\right)+\mathcal{O}\left(\delta^{2}\right)+\mathcal{O}(\gamma)
$$

Rearranging (4.1), and dropping $\mathcal{O}\left(\delta^{2}\right)$ and $\mathcal{O}(\gamma)$ yield

$$
\delta E_{j}^{ \pm(1)}\left(\rho, z_{ \pm}\right)=E_{j}^{ \pm \gamma}\left(\rho, z_{ \pm}\right)-E_{j}^{ \pm(0)}\left(\rho, z_{ \pm}\right)
$$

which is the linearization of the nonlinear inverse problem and enables us to find an explicit reconstruction formula for the linearized inverse problem.

Noting $\phi=\delta \psi$ and thus $\phi_{n}=\delta \psi_{n}$, where $\phi_{n}$ is the Fourier coefficient of $\phi$. Plugging (3.53) into (4.2), we may deduce that

$$
\phi_{n}=C_{j n}^{-1}\left[E_{j n}^{ \pm \gamma}\left(z_{ \pm}\right)-E_{j n}^{ \pm(0)}\left(z_{ \pm}\right)\right] e^{\mp \mathrm{i} \beta_{n}^{ \pm} z_{ \pm}},
$$

where $E_{j n}^{ \pm \gamma}\left(z_{ \pm}\right)$is the Fourier coefficient of the noisy data $E_{j}^{ \pm \gamma}\left(\rho, z_{ \pm}\right)$and $E_{j n}^{ \pm(0)}\left(z_{ \pm}\right)$is the Fourier coefficient of $E_{j}^{ \pm(0)}\left(\rho, z_{ \pm}\right)$given as

$$
E_{j n}^{+(0)}\left(z_{+}\right)=p_{j}\left(e^{-\mathrm{i} \kappa_{+} z_{+}}+r e^{\mathrm{i} \kappa_{+} z_{+}}\right) \delta_{0 n} \quad \text { and } \quad E_{j n}^{-(0)}\left(z_{-}\right)=p_{j} t e^{-\mathrm{i} \kappa_{-} z_{-}} \delta_{0 n} .
$$

Here $\delta_{0 n}$ the Kronecker's delta function.

It follows from (4.3) and the definitions of $\beta_{n}^{ \pm}$in (2.6), (2.9) that it is well-posed to reconstruct those Fourier coefficients $\phi_{n}$ with $\left|\alpha_{n}\right|<\kappa_{ \pm}$, since the small variations of the measured data will not be amplified and lead to large errors in the reconstruction, but the resolution of the reconstructed function $f$ is restricted by the given wavenumber $\kappa_{ \pm}$. In contrast, it is severely ill-posed to reconstruct those Fourier coefficients $\phi_{n}$ with $\left|\alpha_{n}\right|>\kappa_{ \pm}$, since the small variations in the data will be exponentially enlarged and lead to huge errors in the reconstruction, but they contribute to the super resolution of the reconstructed function $\phi$.

To obtain a stable and super-resolved reconstruction, we adopt a regularization to suppress the exponential growth of the reconstruction errors. Besides, we may use as small $\left|z_{ \pm}\right|$as possible, i.e., measure the data at the distance which is as close as possible to the grating surface which is exactly the idea of near-field optics.

We consider the spectral cut-off regularization. Define the signal-to-noise ratio (SNR) by

$$
\mathrm{SNR}=\min \left\{\delta^{-2}, \gamma^{-1}\right\}
$$

For fixed $z_{ \pm}$, the cut-off frequency $\omega_{ \pm}$is chosen in such a way that

$$
e^{\left|z_{ \pm}\right|\left(\omega_{ \pm}^{2}-\kappa_{ \pm}^{2}\right)^{1 / 2}}=\mathrm{SNR},
$$

which implies that the spatial frequency will be cut-off for those below the noise level. More explicitly, we have

$$
\frac{\omega_{ \pm}}{\kappa_{ \pm}}=\left[1+\left(\frac{\log \mathrm{SNR}}{\kappa_{ \pm}\left|z_{ \pm}\right|}\right)^{2}\right]^{1 / 2},
$$

which indicates $\omega_{ \pm}>\kappa_{ \pm}$as long as SNR $>0$ and super resolution may be achieved.

Taking into account the frequency cut-off, we may have a regularized reconstruction formulation for (4.3):

$$
\phi_{n}=C_{j n}^{-1}\left[E_{j n}^{ \pm \gamma}\left(z_{ \pm}\right)-E_{j n}^{ \pm(0)}\left(z_{ \pm}\right)\right] e^{\mp \mathrm{i} \beta_{n}^{ \pm} z_{ \pm}} \chi_{n}^{ \pm},
$$



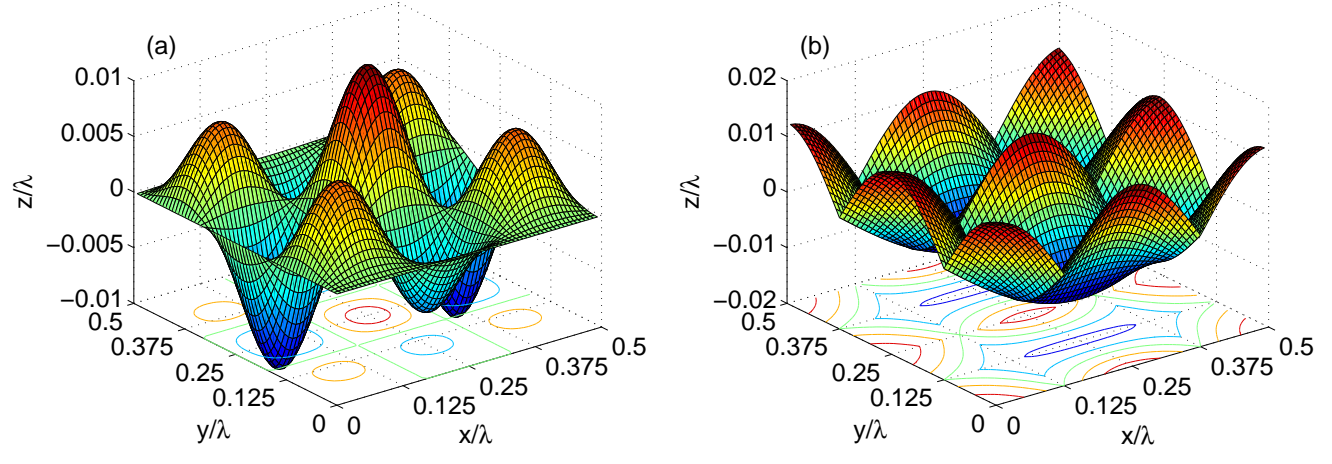

FiguRE 2. The exact grating profile $\psi$. (a) Example 1: smooth grating profile with finitely many Fourier modes; (b) Example 2: non-smooth grating profile with infinitely many Fourier modes.

where the characteristic function

$$
\chi_{n}^{ \pm}=\left\{\begin{array}{lll}
1 & \text { for } & \left|\alpha_{n}\right| \leq \omega_{ \pm} \\
0 & \text { for } & \left|\alpha_{n}\right|>\omega_{ \pm}
\end{array}\right.
$$

Once $\phi_{n}$ are computed, the grating surface function can be approximated by

$$
\begin{aligned}
\phi(\rho) & \approx \sum_{n \in \mathbb{Z}} \phi_{n} e^{\mathrm{i} \alpha_{n} \cdot \rho}=\sum_{\left|\alpha_{n}\right| \leq \omega_{ \pm}} C_{j n}^{-1}\left[E_{j n}^{ \pm \gamma}\left(z_{ \pm}\right)-E_{j n}^{ \pm(0)}\left(z_{ \pm}\right)\right] e^{\mathrm{i}\left(\alpha_{n} \cdot \rho \mp \beta_{n}^{ \pm} z_{ \pm}\right)} \\
& =\sum_{\left|\alpha_{n}\right| \leq \omega_{ \pm}} C_{j n}^{-1} E_{j n}^{ \pm \gamma}\left(z_{ \pm}\right) e^{\mathrm{i}\left(\alpha_{n} \cdot \rho \mp \beta_{n}^{ \pm} z_{ \pm}\right)}-\sum_{\left|\alpha_{n}\right| \leq \omega_{ \pm}} C_{j n}^{-1} E_{j n}^{ \pm(0)}\left(z_{ \pm}\right) e^{\mathrm{i}\left(\alpha_{n} \cdot \rho \mp \beta_{n}^{ \pm} z_{ \pm}\right)} .
\end{aligned}
$$

Substituting (4.4) into (4.5), we obtain an reconstructed grating surface function

$$
\phi(\rho) \approx \sum_{\left|\alpha_{n}\right| \leq \omega_{ \pm}} C_{j n}^{-1} E_{j n}^{ \pm \gamma}\left(z_{ \pm}\right) e^{\mathrm{i}\left(\alpha_{n} \cdot \rho \mp \beta_{n}^{ \pm} z_{ \pm}\right)}-C_{j 0}^{-1}\left(r+e^{-2 \mathrm{i} \kappa_{+} z_{+}}\right) p_{j}
$$

from the reflection configuration or

$$
\phi(\rho) \approx \sum_{\left|\alpha_{n}\right| \leq \omega_{ \pm}} C_{j n}^{-1} E_{j n}^{ \pm \gamma}\left(z_{ \pm}\right) e^{\mathrm{i}\left(\alpha_{n} \cdot \rho \mp \beta_{n}^{ \pm} z_{ \pm}\right)}-C_{j 0}^{-1} t p_{j}
$$

from the transmission configuration.

Hence, only two fast Fourier transforms are needed to reconstruct the grating surface function: one is done for the data to obtain $E_{j n}^{ \pm \gamma}\left(z_{ \pm}\right)$and another is done to obtain the approximated function $\phi$.

\section{NUMERICAL EXPERIMENT}

In this section, we discuss the algorithmic implementation for the direct and inverse problems and present two numerical examples to illustrate the effectiveness of the proposed method. As is shown in Fig. 2, two types of grating profiles are considered. One is a smooth function with finitely many Fourier modes and another is a non-smooth function with infinitely many Fourier modes. Although the method requires $\psi \in C^{2}\left(\mathbb{R}^{2}\right)$, it is applicable to non-smooth functions numerically.

The second-order Nédélec edge element is adopted to solve the direct problem and obtain the synthetic scattering data. Uniaxial perfect matched layer (PML) boundary condition is imposed on the $z$ direction to truncated the domain. An adaptive mesh refinement technique [15] is used to achieve the solution with a specified accuracy in an optimal fashion. Our implementation is based on parallel hierarchical grid (PHG) [43], which is a toolbox for developing parallel adaptive finite element programs on unstructured tetrahedral 
meshes. To have a tetrahedral mesh with biperiodic boundary points, we generate an uniform hexahedral mesh and then divide each hexahedron into six tetrahedrons. The linear system resulted from finite element discretization is solve by the multifrontal massively parallel sparse direct solver [1] .

In the following two examples, the incident wave is taken as $\mathbf{E}^{\mathrm{inc}}=(1,0,0) e^{-\mathrm{i} \kappa_{+} z}$, i.e., $p_{1}=1$ and $p_{2}=p_{3}=0$, and only the first component of the electric field, $E_{1}^{+}(\rho, h)$, needs to be measured. The wavenumber in $\Omega_{S}^{-}$is $\kappa_{-}=1.6 \pi$. The wavenumber in $\Omega_{S}^{+}$is $\kappa_{+}=\pi$, which corresponds to the wavelength $\lambda=2$. Define by $R$ the unit rectangular domain, i.e., $R=[0,0.5 \lambda] \times[0,0.5 \lambda]$. The computational domain is $R \times[-0.3 \lambda, 0.3 \lambda]$ with the PML region $(R \times[-0.3 \lambda,-0.15 \lambda]) \cup(R \times[0.15 \lambda, 0.3 \lambda])$. The scattering data $E_{1}^{+}(\rho, h)$ is obtained by interpolation into the uniform $256 \times 256$ grid points on the measurement plane $z=h$. In all the figures, the plots are rescaled with respect to the wavelength $\lambda$ to clearly show the relative size. The results are plotted on $64 \times 64$ grid points instead of $256 \times 256$ grid points in order to reduce the display sizes. To test the stability of the method, a random noise is added to the scattering data, i.e., the scattering data takes the form

$$
E_{1}^{+\gamma}(\rho, h)=E_{1}^{+}(\rho, h)(1+\gamma \text { rand }),
$$

where rand stands for uniformly distributed random numbers in $[-1,1]$ and $\gamma$ is the noise level parameter. The relative $L^{2}(R)$ error is defined by

$$
e=\frac{\left\|\phi-\phi_{\gamma, \delta}\right\|_{0, R}}{\|\phi\|_{0, R}}
$$

where $\phi$ is the exact surface function and $\phi_{\gamma, \delta}$ is the reconstructed surface function.

Example 1. This example illustrates the reconstruction results of a smooth grating profile with finitely many Fourier modes, as seen in Figure 2 a). The exact grating surface function is given by $\phi(\rho)=\delta \psi(\rho)$, where the grating profile function

$$
\psi(x, y)=0.5 \sin (3 \pi x)(\cos (2 \pi y)-\cos (4 \pi y)) .
$$

First, consider the surface deviation parameter $\delta$. The measurement is taken at $h=0.1 \lambda$ and no additional random noise is added to the scattering data, i.e., $\gamma=0$. This test is to investigate the influence of surface deformation parameter on the reconstructions. In (4.2), higher order terms of $\delta$ are dropped in the power series to linearize the inverse problem and to obtain the explicit reconstruction formulas. As expected, the smaller the surface deformation $\delta$ is, the more accurate is the approximation of the linearized model to the original nonlinear model problem. Table 1 shows the relative $L^{2}(R)$ error of the reconstructions with three different surface deformation parameters $\delta=0.05 \lambda, 0.025 \lambda, 0.0125 \lambda$ for a fixed measurement distance $h=0.1 \lambda$. It is clear to note that the error decreases from $45.3 \%$ to $15.6 \%$ as $\delta$ decreases from $0.05 \lambda$ to $0.0125 \lambda$.

TABLE 1. Example 1: Relative error of the reconstructions by using different $\delta$ with $h=$ $0.1 \lambda$ and $\gamma=0.0$.

\begin{tabular}{llll}
\hline \hline$\delta$ & $0.05 \lambda$ & $0.025 \lambda$ & $0.0125 \lambda$ \\
\hline$e$ & $4.53 \times 10^{-1}$ & $2.49 \times 10^{-1}$ & $1.56 \times 10^{-1}$ \\
\hline \hline
\end{tabular}

Next is to consider the noise level $\gamma$ and the measurement distance $h$. In practice, the scattering data always contains a certain amount of noise. To test the stability and super resolving capability of the method, we add $1 \%$ and $5 \%$ random noises to the scattering data. Table 2 and 3 report the relative $L^{2}(R)$ error of the reconstructions with four different measurement distances $h=0.1 \lambda, 0.075 \lambda, 0.05 \lambda, 0.025 \lambda$ for a fixed $\delta=0.0125 \lambda$. Comparing the results for the same $\delta=0.0125 \lambda$ and $h=0.1 \lambda$ in Tables 1 and 3 , we can see that the relative error increases dramatically from $15.6 \%$ by using noise free data to $83.8 \%$ by using $5 \%$ noise data. The reason is that a smaller cut-off should be chosen to suppress the exponentially increasing noise in the data and thus the Fourier modes of the exact grating surface function can not be recovered for those higher than the cut-off frequency, which leads to a large error and poor resolution in the reconstruction. A smaller measurement distance is desirable in order to have a large cut-off frequency, which enhances the resolution and reduces the error. As can be seen in Table 2, the reconstruction error decreases from $56.7 \%$ by using 

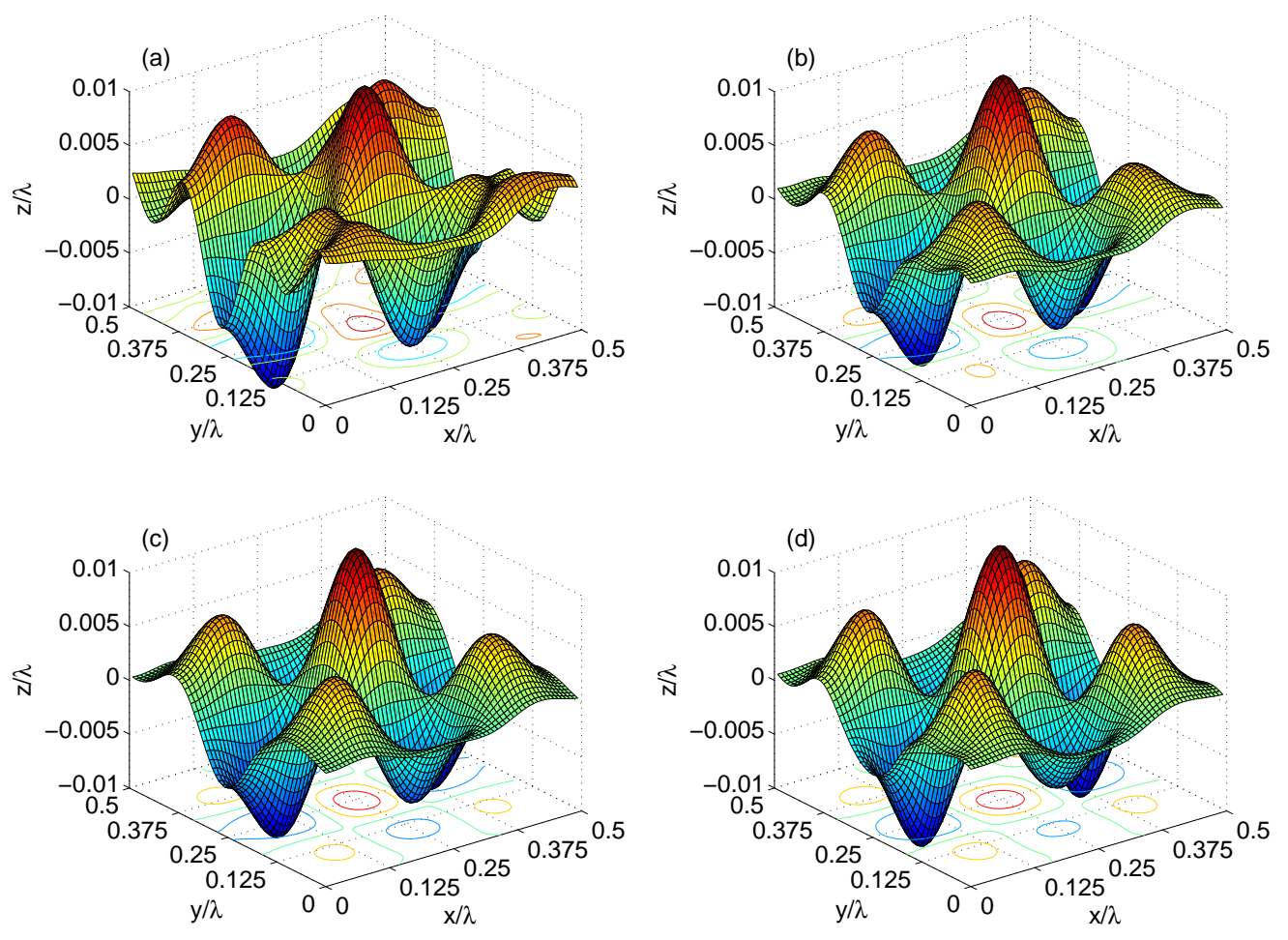

FIGURE 3. Example 1: Reconstructed grating surfaces by using different $h$ with $\delta=$ $0.0125 \lambda$ and $\gamma=1 \%$. (a) $h=0.1 \lambda$; (b) $h=0.075 \lambda$; (c) $h=0.05 \lambda$; (d) $h=0.025 \lambda$.

$h=0.1 \lambda$ to as low as $16.7 \%$ by using $h=0.025 \lambda$ for $1 \%$ noise data. Similarly, in Table 3 , the reconstruction error decreases from $83.8 \%$ by using $h=0.1 \lambda$ to as low as $29.5 \%$ by using $h=0.025 \lambda$ even for $5 \%$ noise data. Figure 3 plots the reconstructed surfaces by using $h=0.1 \lambda, 0.075 \lambda, 0.05 \lambda, 0.025 \lambda$. Comparing the exact surface profile in Fig. 22 a) and the reconstructed surface in Fig. 3(d), we can see that the reconstruction is almost perfect and the difference is little by carefully checking the contour plots.

TABLE 2. Example 1: Relative error of the reconstructions by using different $h$ with $\delta=$ $0.0125 \lambda$ and $\gamma=1 \%$.

\begin{tabular}{lllll}
\hline \hline$h$ & $0.1 \lambda$ & $0.075 \lambda$ & $0.05 \lambda$ & $0.025 \lambda$ \\
\hline$e$ & $5.67 \times 10^{-1}$ & $2.95 \times 10^{-1}$ & $2.08 \times 10^{-1}$ & $1.67 \times 10^{-1}$ \\
\hline \hline
\end{tabular}

TABLE 3. Example 1: Relative error of the reconstructions by using different $h$ with $\delta=$ $0.0125 \lambda$ and $\gamma=5 \%$.

\begin{tabular}{lllll}
\hline \hline$h$ & $0.1 \lambda$ & $0.075 \lambda$ & $0.05 \lambda$ & $0.025 \lambda$ \\
\hline$e$ & $8.38 \times 10^{-1}$ & $8.06 \times 10^{-1}$ & $5.56 \times 10^{-1}$ & $2.95 \times 10^{-1}$ \\
\hline \hline
\end{tabular}

Example 2. This example illustrates the reconstruction results of a non-smooth grating profile with infinitely many Fourier modes, as seen in Fig. 2(b). The exact grating surface function is given by $\phi(\rho)=\delta \psi(\rho)$, where the grating profile function

$$
\psi(x, y)=|\cos (2 \pi x) \cos (2 \pi y)|-|\sin (\pi x) \sin (2 \pi y)| .
$$


First is to consider the influence of $\delta$ by using noise-free data. The measurement is taken at $h=0.1 \lambda$. Table 4 presents the relative $L^{2}(R)$ error of the reconstructions with three different surface deformation parameters $\delta=0.05 \lambda, 0.025 \lambda, 0.0125 \lambda$. The error decreases from $35.8 \%$ to $16.0 \%$ as $\delta$ decreases from $0.05 \lambda$ to $0.0125 \lambda$. Based on these results, the following observation can be made: a smaller deformation parameter $\delta$ yields a better reconstruction.

TABLE 4. Example 2: Relative error of the reconstructions by using different $\delta$ with $h=$ $0.1 \lambda$ and $\gamma=0.0$.

\begin{tabular}{llll}
\hline \hline$\delta$ & $0.05 \lambda$ & $0.025 \lambda$ & $0.0125 \lambda$ \\
\hline$e$ & $3.58 \times 10^{-1}$ & $2.72 \times 10^{-1}$ & $1.60 \times 10^{-1}$ \\
\hline \hline
\end{tabular}

Next is to consider the influence of the noise level $\gamma$ and the measurement distance $h$. We add $1 \%$ and $5 \%$ random noises to the scattering data. Table 5 and 6 report the relative $L^{2}(R)$ error of the reconstructions with four different measurement distances $h=0.1 \lambda, 0.075 \lambda, 0.05 \lambda, 0.025 \lambda$ for a fixed $\delta=0.0125 \lambda$. Comparing the results for the same $\delta=0.0125 \lambda$ and $h=0.1 \lambda$ in Tables 4 and 6 , we can see that the relative error is more than doubled from $16.0 \%$ by using noise-free data to $34.3 \%$ by using $5 \%$ noise data. Again, the reason is that a smaller cut-off is chosen to suppress the exponentially increasing noise in the data and thus higher Fourier modes of the exact grating surface function can not be recovered. A smaller measurement distance helps to enhance the resolution and reduce the error. In Table 5, the reconstruction error decreases from $27.3 \%$ by using $h=0.1 \lambda$ to as low as $17.3 \%$ by using $h=0.025 \lambda$ for $1 \%$ noise data. In Table 6 , the reconstruction error decreases from $34.3 \%$ by using $h=0.1 \lambda$ to as low as $24.4 \%$ by using $h=0.025 \lambda$ for $5 \%$ noise data. Figure 4 shows the reconstructed surfaces by using $h=0.1 \lambda, 0.075 \lambda, 0.05 \lambda, 0.025 \lambda$. Comparing the exact surface profile in Fig. 2(b) and the reconstructed surface in Fig. 4(d), we can see that a good reconstruction can still be obtained when using a small measurement distance.

TABLE 5. Example 2: Relative error of the reconstructions by using different $h$ with $\delta=$ $0.0125 \lambda$ and $\gamma=1 \%$.

\begin{tabular}{lllll}
\hline \hline$h$ & $0.1 \lambda$ & $0.075 \lambda$ & $0.05 \lambda$ & $0.025 \lambda$ \\
\hline$e$ & $2.73 \times 10^{-1}$ & $2.44 \times 10^{-1}$ & $1.88 \times 10^{-1}$ & $1.73 \times 10^{-1}$ \\
\hline \hline
\end{tabular}

TABLE 6. Example 2: Relative error of the reconstructions by using different $h$ with $\delta=$ $0.0125 \lambda$ and $\gamma=5 \%$.

\begin{tabular}{lllll}
\hline \hline$h$ & $0.1 \lambda$ & $0.075 \lambda$ & $0.05 \lambda$ & $0.025 \lambda$ \\
\hline$e$ & $3.43 \times 10^{-1}$ & $2.99 \times 10^{-1}$ & $2.81 \times 10^{-1}$ & $2.44 \times 10^{-1}$ \\
\hline \hline
\end{tabular}

\section{CONCLUSION}

We have presented an effective computational method to reconstruct surfaces of biperiodic dielectric gratings. Subwavelength resolution is achieved stably. Based on the transformed field expansion, an analytic solution is deduced for the direct problem. The nonlinear inverse problem is linearized by dropping higher order terms in power series. Explicit reconstruction formulas are obtained and are implemented by using the FFT. Two representative numerical examples are considered: one is a smooth function which has finitely many Fourier modes and another is a nonsmooth function which has infinitely many Fourier modes. We have carefully investigated the influence of the parameters on the reconstructions. The results show that super resolution may be achieved by using small measurement distance. There are many interesting and challenging mathematical problems, such as uniqueness, stability, resolution, and error estimates, which are remaining and left for future work. We will report the results elsewhere. 

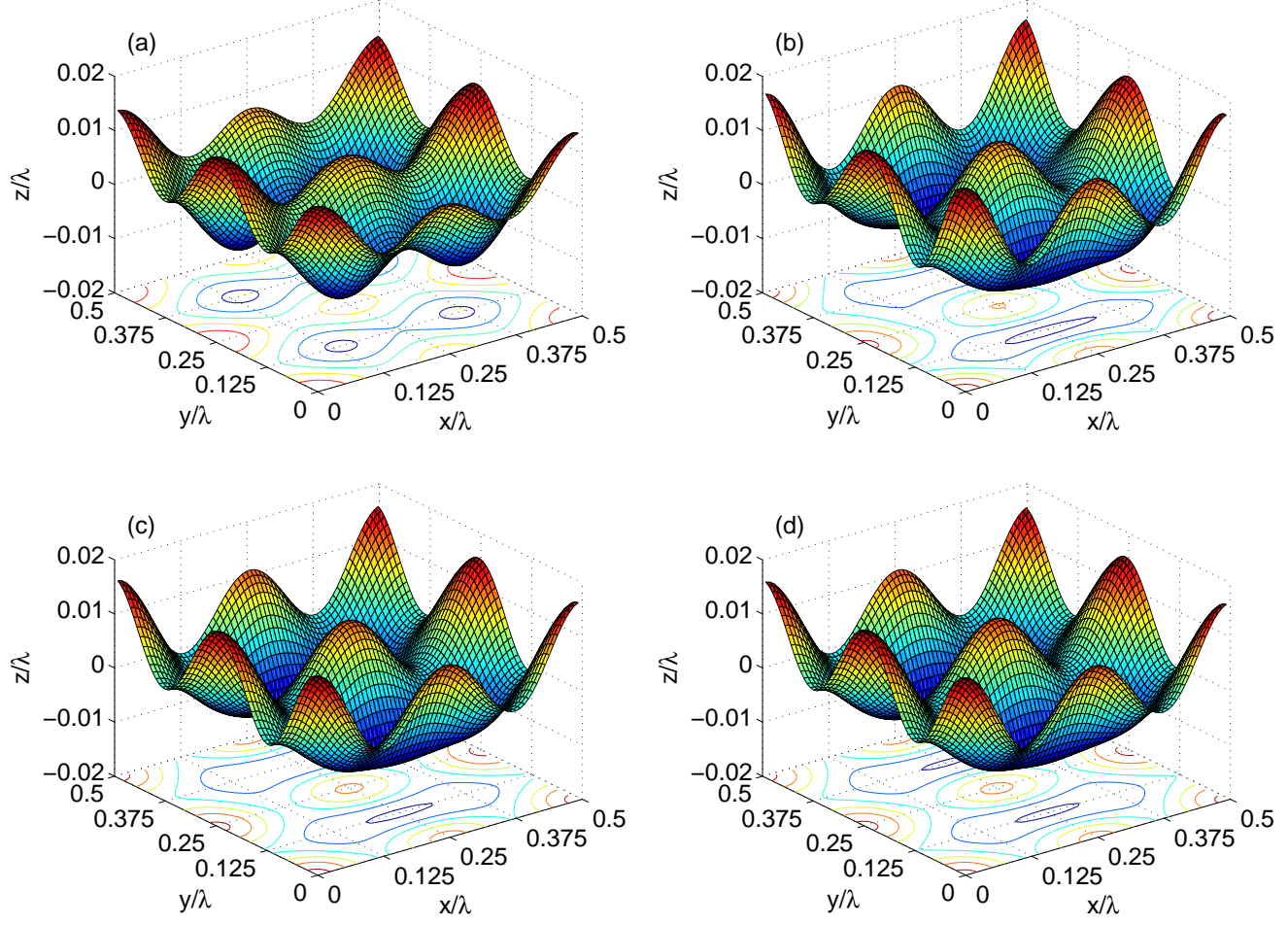

FIGURE 4. Example 2: Reconstructed grating surfaces by using different $h$ with $\delta=$ $0.0125 \lambda$ and $\gamma=1 \%$. (a) $h=0.1 \lambda$; (b) $h=0.075 \lambda$; (c) $h=0.05 \lambda$; (d) $h=0.025 \lambda$.

\section{REFERENCES}

[1] P. R. Amestoy, I. S. Duff, J. Koster, and J.-Y. L'Excellent, A fully asynchronous multifrontal solver using distributed dynamic scheduling, SIAM. J. Matrix Anal. \& Appl., 23 (2001), pp. 15-41.

[2] H. AMmARI, Uniqueness theorems for an inverse problem in a doubly periodic structure, Inverse Problems, 11 (1995), pp. 823-833.

[3] I. AKDUMAN, R. KRESS, AND A. YAPAR, Iterative reconstruction of dielectric rough surface profiles at fixed frequency, Inverse Problems, 22 (2006), pp. 939-954.

[4] T. ARENS AND A. KIRSCH, The factorization method in inverse scattering from periodic structures, Inverse Problems, 19 (2003), pp. 1195-1211.

[5] G. BAO, A unique theorem for an inverse problem in periodic diffractive optics, Inverse Problems, 10 (1994), pp. 335-340.

[6] G. BAO, Variational approximation of Maxwell's equations in biperiodic structures, SIAM J. Appl. Math., 57 (1997), pp. 364-381.

[7] G. Bao, L. Cowsar, And W. Masters, Mathematical Modeling in Optical Science, Frontiers Appl. Math., 22, SIAM, Philadelphia, 2001.

[8] G. BAO, T. CUI, AND P. LI, Inverse diffraction grating of Maxwell's equations in biperiodic structures, Optics Express, 22 (2014), pp. 4799-4816.

[9] G. BAo, D. Dobson, And J. A. Cox, Mathematical studies in rigorous grating theory, J. Opt. Soc. Amer. A, 12 (1995), pp. 1029-1042.

[10] G. BAO AND A. FRIEDMAN, Inverse problems for scattering by periodic structure, Arch. Rational Mech. Anal., 132 (1995), pp. 49-72.

[11] G. BAO AND P. LI, Near-field imaging of infinite rough surfaces, SIAM J. Appl. Math., 73 (2013), pp. $2162-2187$.

[12] G. BAO AND P. LI, Near-field imaging of infinite rough surfaces in dielectric media, SIAM J. Imaging Sci., 7 (2014), pp. 867-899.

[13] G. BAO AND P. LI, Convergence analysis in near-field imaging, Inverse Problems, 30 (2014), pp. 085008.

[14] G. BAO AND J. Lin, Near-field imaging of the surface displacement on an infinite ground plane, Inverse Probl. Imag., 7 (2013), pp. 377-396.

[15] G. BAO, P. LI, AND H. WU, An adaptive edge element method with perfectly matched absorbing layers for wave scattering by biperiodic structures, Math. Comp., 79 (2009), pp. 1-34. 
[16] G. BAO, P. LI, AND H. Wu, A computational inverse diffraction grating problem, J. Opt. Soc. Am. A, 29 (2012), pp. $394-399$.

[17] G. BAO, P. Li, AND J. Lv, Numerical solution of an inverse diffraction grating problem from phasless data, J. Opt. Soc. Am. A, 30 (2013), pp. 293-299.

[18] G. BAO, H. ZHANG, AND J. ZOU, Unique determination of periodic polyhedral structures by scattered electromagnetic fields, Trans. Amer. Math. Soc., 363 (2011), pp. 4527-4551.

[19] G. BAO AND Z. ZHOU, An inverse problem for scattering by a doubly periodic structure, Trans. Amer. Math. Soc., 350 (1998), 4089-4103.

[20] G. Bruckner, J. Cheng, And M. Yamamoto, An inverse problem in diffractive optics: conditional stability, Inverse Problems, 18 (2002), pp. 415-433.

[21] G. BRUCKNER AND J. ELSCHNER, A two-step algorithm for the reconstruction of perfectly reflecting periodic profiles, Inverse Problems, 19 (2003), pp. 315-329.

[22] O. BRUNO AND F. ReITICH, Numerical solution of diffraction problems: a method of variation of boundaries, J. Opt. Soc. Am. A, 10 (1993), pp. 1168-1175.

[23] S. CARNEY AND J. SCHOTlAnd, Inverse scattering for near-field microscopy, App. Phys. Lett., 77 (2000), pp. 2798-2800.

[24] S. CARney And J. Schotland, Near-field tomography, MSRI Ser. Math. Appl., 47 (2003), pp. 133-168.

[25] T. Cheng, P. Li, and Y. Wang, Near-field imaging of perfectly conducting grating surfaces, J. Opt. Soc. Am. A, 30 (2013), pp. 2473-2481.

[26] R. Coifman, M. Goldberg, T. HRycAK, M. IsRAeli, AND V. RokHLin, An improved operator expansion algorithm for direct and inverse scattering computations, Waves Random Media, 9 (1999), pp. 441-457.

[27] D. CourJon, Near-Field Microscopy and Near-Field Optics, Imperial College Press, London, 2003.

[28] J. A. DES ANTO AND R. J. WombelL, The reconstruction of shallow rough-surface profiles from scattered field data, Inverse Problems, 7 (1991), pp. L7-L12.

[29] D. Dobson, A variational method for electromagnetic diffraction in biperiodic structures, Math. Model. Numer. Anal., 28 (1994), pp. 419-439.

[30] J. Elschner, G. Hsiao, And A. Rathsfeld, Grating profile reconstruction based on finite elements and optimization techniques, SIAM J. Appl. Math., 64 (2003), pp. 525-545.

[31] F. HetTlich, Iterative regularization schemes in inverse scattering by periodic structures, Inverse Problems, 18 (2002), pp. 701-714.

[32] F. Hettlich AND A. KIRSCH, Schiffer's theorem in inverse scattering theory for periodic structures, Inverse Problems, 13 (1997), pp. 351-361.

[33] G. HU, J. YANG, AND B. ZHANG, An inverse electromagnetic scattering problem for a bi-periodic inhomogeneous layer on a perfectly conducting plate, Appl. Anal., 90 (2011), 317-333.

[34] G. HU AND B. ZHANG, The linear sampling method for inverse electromagnetic scattering by a partially coated bi-periodic structures, Math. Meth. Appl. Sci., 34 (2011), pp. 509-519.

[35] K. ITO AND F. REITICH, A high-order perturbation approach to profile reconstruction: I. Perfectly conducting gratings, Inverse Problems, 15 (1999), pp. 1067-1085.

[36] A. KIRSCH, Uniqueness theorems in inverse scattering theory for periodic structures, Inverse Problems, 10 (1994), pp. 145152.

[37] R. KRESS AND T. TRAN, Inverse scattering for a locally perturbed half-plane, Inverse Problems, 16 (2000), pp. 1541-1559.

[38] A. LeChleiter AND D. L. NGUYen, On uniqueness in electromagnetic scattering from biperiodic structures, ESAIM: M2AN, 47 (2013), pp. 1167-1184.

[39] A. LECHLEITER AND D. L. NGUYEN, Factorization method for electromagnetic inverse scattering from biperiodic structures, SIAM J. Imaging Sci., 6 (2013), pp. 1111-1139.

[40] J. C. NÉDÉLEC AND F. STARLING, Integral equation methods in a quasi-periodic diffraction problem for the time-harmonic Maxwell's equations, SIAM J. Math. Anal., 22 (1991), pp. 1679-1701.

[41] D. P. NiCHOLls AND F. REITICH, Shape deformations in rough surface scattering: cancellations, conditioning, and convergence, J. Opt. Soc. Am. A, 21 (2004), pp. 590-605.

[42] R. Petit, ed., Electromagnetic Theory of Gratings, Springer-Verlag, 1980.

[43] PHG (Parallel Hierarchical Grid), http://1sec.cc.ac.cn/phg/.

[44] J. YANG AND B. ZHANG, Inverse electromagnetic scattering problems by a doubly periodic structure, Math. Appl. Anal., 18 (2011), pp. 111-126.

School of Science, Beijing University of Posts and Telecommunications, Beijing 100876, China.

E-mail address: jxue@lsec.cc.ac.cn

Department of Mathematics, Purdue University, West Lafayette, Indiana 47907, USA.

E-mail address: lipeijun@math.purdue.edu 